\title{
Inspection plan for COVID-19 patients for Weibull distribution using repetitive sampling under indeterminacy
}

\author{
G. Srinivasa Rao ${ }^{1}$ and Muhammad Aslam ${ }^{2^{*}}$ (D)
}

\begin{abstract}
Background: This research work is elaborated investigation of COVID-19 data for Weibull distribution under indeterminacy using time truncated repetitive sampling plan. The proposed design parameters like sample size, acceptance sample number and rejection sample number are obtained for known indeterminacy parameter.

Methods: The plan parameters and corresponding tables are developed for specified indeterminacy parametric values. The conclusion from the outcome of the proposed design is that when indeterminacy values increase the average sample number (ASN) reduces.

Results: The proposed repetitive sampling plan methodology application is given using COVID-19 data belong to Italy. The efficiency of the proposed sampling plan is compared with the existing sampling plans.

Conclusions: Using the tables and COVID-19 data illustration, it is concluded that the proposed plan required a smaller sample size as examined with the available sampling plans in the literature.
\end{abstract}

Keywords: Repetitive sampling plan, Traditional statistics, Indeterminacy, COVID-19, Average sample number

\section{Background}

It is broadly established that a huge number of COVID19 cases are unnoticed worldwide. A rudimentary measure of population occurrence is the small part of positive cases for a given date in any country. On the other hand, this is subject to largely found that bias since tests are normally only ordered from suggestive cases, whereas a large proportion of infected people might show little symptoms or sometimes no symptoms for more details see [1]. Most governments are applying the mechanism of test randomly selected individuals to estimate the true disease occurrence in inhabitants in a particular locality. Nevertheless, when the disease occurrence is low and difficult to acquire from each patient/person by tests,

\footnotetext{
* Correspondence: aslam_ravian@hotmail.com

${ }^{2}$ Department of Statistics, Faculty of Science, King Abdulaziz University, Jeddah 21551, Saudi Arabia

Full list of author information is available at the end of the article
}

under such situations we may use an acceptance sampling plan under indeterminacy. The health practitioners are paying attention to estimate the average number of deaths or ratio of deaths to the total number of COVID19 death cases on daily basis, for the coming days, next week or month, etc. Reference [2]. In such a case, the health practitioners are paying attention to test the null hypothesis that the average number of deaths or ratio of deaths to the total number of COVID-19 death cases on daily basis is equal to the specified average number of deaths due to COVID-19 against the alternative hypothesis that the average number of deaths due to COVID19 varies significantly. In this situation for testing of the hypothesis, practically it is difficult to record the average number of death for the whole year, whereas it is easy to record the daily basis and the average number of deaths can be obtained from the randomly selected days. The null hypothesis may be rejected if the daily average

C C The Author(s). 2021 Open Access This article is licensed under a Creative Commons Attribution 4.0 International License, which permits use, sharing, adaptation, distribution and reproduction in any medium or format, as long as you give appropriate credit to the original author(s) and the source, provide a link to the Creative Commons licence, and indicate if changes were made. The images or other third party material in this article are included in the article's Creative Commons licence, unless indicated otherwise in a credit line to the material. If material is not included in the article's Creative Commons licence and your intended use is not permitted by statutory regulation or exceeds the permitted use, you will need to obtain permission directly from the copyright holder. To view a copy of this licence, visit http://creativecommons.org/licenses/by/4.0/ The Creative Commons Public Domain Dedication waiver (http://creativecommons.org/publicdomain/zero/1.0/) applies to the data made available in this article, unless otherwise stated in a credit line to the data. 
number of deaths due to COVID-19, state acceptance number of days, is more than or equal to the specified average number of deaths due to COVID-19 throughout the given number of days.

Many researchers have done studies on the time truncated life test for various distributions. Some of them are [3] developed the acceptance sampling plan for life tests: log-logistic models. Reference [4] derived acceptance sampling based on truncated life tests for generalized Rayleigh distribution. Reference [5] developed the acceptance sampling plans based on the generalized Birnbaum-Saunders distribution. Reference $[6,7]$ constructed the acceptance sampling plans for BirnbaumSaunders and Burr XII distributions. References [8, 9] constructed acceptance sampling plans for extended exponential and generalized inverted exponential distributions. The details about the acceptance sampling plans can be seen in $[10,11]$. The generalization of a single acceptance sampling plan namely repetitive sampling plan, [12] derived the decision rule of the repetitive acceptance sampling plan. The method of repetitive group acceptance sampling plan (RGASP) was first proposed by [13] for an attribute. Reference [14, 15] constructed the RASP for inverse Gaussian distribution and Burr type XII. Reference [16] developed generalized inverted exponential distributions. References [17-19] studied the repetitive sampling plan under different situations.

More details about the neutrosophic logic, their measure of determinacy, and indeterminacy are given by [20]. Numerous authors studied the neutrosophic logic for various real problems and showed its efficiency over fuzzy logic, for more details refer [21-26]. The idea of neutrosophic statistics was given using the idea of neutrosophic logic, [27-29]. The neutrosophic statistics give information about the measure of determinacy and measure of indeterminacy, see [30]. The neutrosophic statistics reduce to classical statistics if no information is recorded about the measure of indeterminacy. References [31-33] proposed the acceptance sampling plans using neutrosophic statistics [34]. proposed the timetruncated group plans for the Weibull distribution. Reference [35] worked on neutrosophic Weibull and neutrosophic family of Weibull distribution.

The existing sampling plans based on classical statistics and fuzzy philosophies do not give information about the measure of indeterminacy. Reference [36] worked on the single sampling plan using a fuzzy approach. Reference [37] discussed the effect of sampling error on inspection using a fuzzy approach. Reference [38] proposed a single plan using fuzzy logic. Reference [39] proposed the improved sampling plan using fuzzy logic. For details, the reader may refer to $[40,41]$. To the best of our knowledge, there is no work on a timetruncated sampling plan for Weibull distribution under indeterminacy. In this paper, a repetitive acceptance sampling plan for Weibull distribution under indeterminacy is developed to testing the daily average deaths. We are anticipated the proposed sampling plan shows a fewer sample size as compared with the existing sampling plans for testing the daily average deaths.

In Section 2, we present an introduction of a repetitive acceptance sampling plan for Weibull distribution under indeterminacy. In Section 3, the proposed repetitive acceptance sampling plan under indeterminacy is compared with the single sampling plan proposed by [42] The proposed sampling plan is illustrated using COVID19 data belong to Italy, which was recorded from 1 April to 20 July 2020 in Section 4. Finally, the conclusions and future research works are established in Section 5.

\section{Methods}

The repetitive acceptance sampling plan depends upon the truncated life test procedure is developed by [4345]. The operational steps of this test are given as follows:

Step 1: Draw a sample of size $n$ from the lot. These samples can be put on a life test for a fixed time $t_{0}$. Specify the average $\mu_{0}$ and indeterminacy parameter $I_{N} \in\left[I_{L}, I_{U}\right]$.

Step 2: Accept $H_{0}: \mu_{N}=\mu_{0 N}$ if the daily average deaths in $c_{1}$ days are more than or equal to $\mu_{0}$ (i.e., $\mu_{0} \leq c_{1}$ ). If daily average deaths in $c_{2}$ days are less than to $\mu_{0}$ (i.e., $\mu_{0}>c_{2}$ ) then we reject $H_{0}: \mu_{N}=\mu_{0 N}$ and terminate the test, where $c_{1 \leq} c_{2}$.

Step 3: If $c_{1}<\mu_{0} \leq c_{2}$ then go to step 1 and repeat the above experiment.

The above procedure of repetitive acceptance sampling plan (RASP) mainly depends on four characteristics those are $n, c_{1}, c_{2}$ and $I_{N}$, where $I_{N} \epsilon\left[I_{L}, I_{U}\right]$ is considered as the specified parameter and set according to the uncertainty level. RASP is nothing but the generalization of an ordinary single sampling plan under uncertainty. If $c_{1}=c_{2}$ in RASP, it ultimately reduces to a single sampling plan under uncertainty. Suppose that $t_{0}=a \mu_{0}$ be the time in days, where $a$ is the termination ratio. The lot acceptance probability is to be determined with the help of operating characteristic (OC) function for details see [13] and it is given by

$$
L(p)=\frac{P_{a}(p)}{P_{a}(p)+P_{r}(p)} ; 0
$$

Here $P_{a}(p)$ is the probability of accepting $H_{0}: \mu_{N}=\mu_{0 N}$ and $P_{r}(p)$ is the probability of rejecting $H_{0}: \mu_{N}=\mu_{0 N}$, which are given by 


$$
P_{a}(p)=\sum_{i=0}^{c_{1}}\left(\begin{array}{c}
n \\
i
\end{array}\right) p^{i}(1-p)^{n-i}
$$

and

$$
P_{r}(p)=1-\sum_{i=0}^{c_{2}}\left(\begin{array}{c}
n \\
i
\end{array}\right) p^{i}(1-p)^{n-i}
$$

where $\mathrm{p}$ is the probability of unreliability.

Therefore eq. (1) becomes

$$
L(p)=\frac{\sum_{i=0}^{c_{1}}\left(\begin{array}{c}
n \\
i
\end{array}\right) p^{i}(1-p)^{n-i}}{\sum_{i=0}^{c_{1}}\left(\begin{array}{c}
n \\
i
\end{array}\right) p^{i}(1-p)^{n-i}+1-\sum_{i=0}^{c_{2}}\left(\begin{array}{c}
n \\
i
\end{array}\right) p^{i}(1-p)^{n-i}} ; 0
$$

The Weibull distribution under neutrosophic statistics is developed by [42] for the design of the sampling scheme plan for testing the average wind speed under an indeterminate environment.

Suppose that $f\left(x_{N}\right)=f\left(x_{L}\right)+f\left(x_{U}\right) I_{N} ; I_{N} \in\left[I_{L}, I_{U}\right]$ be a neutrosophic probability density function (npdf) having determinate part $f\left(x_{L}\right)$, indeterminate part $f\left(x_{U}\right) I_{N}$ and indeterminacy interval $I_{N} \epsilon\left[I_{L}, I_{U}\right]$. Note that $x_{N} \epsilon\left[x_{L}, x_{U}\right]$ be a neutrosophic random variable follows the npdf. The npdf is the generalization of pdf under classical statistics. The proposed neutrosophic form of $f\left(x_{N}\right) \epsilon\left[f\left(x_{L}\right), f\left(x_{U}\right)\right]$ reduces to pdf under classical statistics when $I_{L}=0$. Based on this information, the npdf of the Weibull dis-

\begin{tabular}{|c|c|c|c|c|c|c|c|c|c|c|c|c|c|c|c|c|c|}
\hline \multirow[t]{2}{*}{$\tilde{\tilde{\beta}}$} & \multirow[t]{2}{*}{$\frac{\mu_{N}}{\mu_{0 N}}$} & \multicolumn{4}{|c|}{$I_{U}=0.00$} & \multicolumn{4}{|c|}{$I_{U}=0.02$} & \multicolumn{4}{|c|}{$I_{U}=0.04$} & \multicolumn{4}{|c|}{$I_{U}=0.05$} \\
\hline & & $\mathrm{n}$ & $c_{1}$ & $c_{2}$ & ASN & $n$ & $c_{1}$ & $c_{2}$ & ASN & $\bar{n}$ & $c_{1}$ & $c_{2}$ & ASN & $\bar{n}$ & $c_{1}$ & $c_{2}$ & ASN \\
\hline 0.25 & 1.1 & 366 & 131 & 149 & 996.01 & 351 & 130 & 148 & 970.30 & 305 & 116 & 134 & 933.78 & 233 & 87 & 107 & 1123.61 \\
\hline 0.25 & 1.2 & 180 & 64 & 70 & 262.79 & 144 & 52 & 59 & 244.14 & 163 & 62 & 68 & 241.62 & 116 & 43 & 51 & 236.76 \\
\hline 0.25 & 1.3 & 67 & 21 & 27 & 135.08 & 71 & 24 & 29 & 121.47 & 57 & 19 & 25 & 124.91 & 81 & 30 & 34 & 117.39 \\
\hline 0.25 & 1.4 & 61 & 20 & 23 & 83.75 & 47 & 15 & 19 & 79.12 & 31 & 9 & 14 & 78.47 & 33 & 10 & 15 & 79.74 \\
\hline 0.25 & 1.5 & 46 & 14 & 17 & 65.19 & 26 & 7 & 11 & 56.28 & 25 & 7 & 11 & 55.12 & 31 & 10 & 13 & 49.83 \\
\hline 0.25 & 1.8 & 30 & 9 & 10 & 34.14 & 24 & 7 & 9 & 33.65 & 18 & 5 & 7 & 26.81 & 27 & 9 & 10 & 31.03 \\
\hline 0.25 & 2.0 & 24 & 7 & 8 & 27.96 & 21 & 6 & 7 & 24.49 & 13 & 3 & 5 & 20.91 & 20 & 6 & 7 & 23.32 \\
\hline 0.10 & 1.1 & - & - & - & - & - & - & - & - & - & - & - & - & - & - & - & - \\
\hline 0.10 & 1.2 & 195 & 65 & 77 & 388.69 & 233 & 83 & 93 & 365.45 & 220 & 81 & 91 & 349.86 & 185 & 68 & 79 & 341.30 \\
\hline 0.10 & 1.3 & 114 & 36 & 44 & 197.37 & 102 & 33 & 41 & 184.31 & 104 & 35 & 43 & 181.35 & 89 & 30 & 38 & 171.55 \\
\hline 0.10 & 1.4 & 74 & 22 & 28 & 121.14 & 81 & 26 & 31 & 115.70 & 69 & 22 & 28 & 113.54 & 65 & 21 & 27 & 110.40 \\
\hline 0.10 & 1.5 & 64 & 19 & 23 & 87.09 & 42 & 11 & 17 & 86.67 & 29 & 7 & 13 & 83.12 & 46 & 14 & 19 & 79.02 \\
\hline 0.10 & 1.8 & 32 & 8 & 11 & 44.72 & 31 & 8 & 11 & 43.09 & 30 & 8 & 11 & 41.58 & 16 & 3 & 7 & 38.95 \\
\hline 0.10 & 2.0 & 23 & 5 & 8 & 35.50 & 26 & 6 & 9 & 36.49 & 22 & 5 & 8 & 32.58 & 18 & 4 & 7 & 30.19 \\
\hline 0.05 & 1.1 & - & - & - & - & - & - & - & - & - & - & - & - & - & - & - & - \\
\hline 0.05 & 1.2 & 292 & 99 & 112 & 454.10 & 254 & 88 & 102 & 440.72 & 230 & 82 & 96 & 417.79 & 250 & 92 & 105 & 406.48 \\
\hline 0.05 & 1.3 & 154 & 49 & 58 & 231.40 & 117 & 37 & 47 & 217.69 & 113 & 37 & 47 & 211.21 & 114 & 38 & 48 & 206.78 \\
\hline 0.05 & 1.4 & 78 & 22 & 30 & 144.10 & 91 & 28 & 35 & 137.49 & 91 & 29 & 36 & 133.57 & 89 & 29 & 36 & 133.17 \\
\hline 0.05 & 1.5 & 55 & 14 & 21 & 103.05 & 44 & 11 & 18 & 97.99 & 61 & 18 & 24 & 93.72 & 57 & 17 & 23 & 90.86 \\
\hline 0.05 & 1.8 & 30 & 6 & 11 & 53.55 & 40 & 10 & 14 & 53.72 & 35 & 9 & 13 & 50.20 & 24 & 5 & 10 & 49.66 \\
\hline 0.05 & 2.0 & 29 & 6 & 10 & 44.08 & 30 & 7 & 10 & 38.81 & 24 & 5 & 9 & 38.60 & 32 & 8 & 11 & 39.32 \\
\hline 0.01 & 1.1 & - & - & - & - & - & - & - & - & - & - & - & - & - & - & - & - \\
\hline 0.01 & 1.2 & 393 & 130 & 150 & 586.50 & 371 & 127 & 147 & 565.50 & 357 & 127 & 146 & 530.22 & 343 & 124 & 143 & 520.89 \\
\hline 0.01 & 1.3 & 191 & 58 & 72 & 290.60 & 196 & 62 & 76 & 283.30 & 178 & 58 & 72 & 271.54 & 187 & 63 & 76 & 262.20 \\
\hline 0.01 & 1.4 & 141 & 41 & 51 & 185.60 & 111 & 32 & 43 & 174.80 & 116 & 35 & 46 & 170.80 & 108 & 33 & 44 & 168.20 \\
\hline 0.01 & 1.5 & 91 & 24 & 33 & 131.10 & 91 & 25 & 34 & 127.60 & 76 & 21 & 30 & 117.90 & 89 & 26 & 35 & 123.50 \\
\hline 0.01 & 1.8 & 50 & 11 & 17 & 67.53 & 45 & 10 & 16 & 62.85 & 39 & 8 & 15 & 62.79 & 46 & 11 & 17 & 61.25 \\
\hline 0.01 & 2.0 & 40 & 8 & 13 & 51.86 & 30 & 5 & 11 & 49.49 & 32 & 6 & 12 & 49.98 & 37 & 8 & 13 & 46.90 \\
\hline
\end{tabular}
tribution is defined as follows.

Table 1 The plan parameter when $\tilde{a}=0.10 ; \beta=1$ and $a=0.50$ 


$$
f\left(x_{N}\right)=\left\{\left(\frac{\beta}{\alpha}\right)\left(\frac{x_{N}}{\alpha}\right)^{\beta-1} e^{-\left(\frac{x N}{\alpha}\right)^{\beta}}\right\}+\left\{\left(\frac{\beta}{\alpha}\right)\left(\frac{x_{N}}{\alpha}\right)^{\beta-1} e^{-\left(\frac{x N}{\alpha}\right)^{\beta}}\right\} I_{N} ; I_{N} \in\left[I_{L}, I_{U}\right]
$$

where $\alpha$ and $\beta$ are scale and shape parameters, respectively. Note here that the proposed npdf of the Weibull distribution is the generalization of pdf of the Weibull distribution under classical statistics. The neutrosophic form of the npdf of the Weibull distribution reduces to the Weibull distribution when $I_{L}=0$. The neutrosophic cumulative distribution function (ncdf) of the Weibull distribution is given by

$$
F\left(x_{N}\right)=1-\left\{e^{-\left(\frac{x_{N}}{\alpha}\right)^{\beta}}\left(1+I_{N}\right)\right\}+I_{N} ; I_{N} \epsilon\left[I_{L}, I_{U}\right]
$$

The neutrosophic mean of the Weibull distribution is given by.

$$
\mu_{N}=\alpha \Gamma(1+1 / \beta)\left(1+I_{N}\right) ; I_{N} \epsilon\left[I_{L}, I_{U}\right]
$$

The null and alternative hypotheses for the daily average deaths are stated as follows:

$$
H_{0}: \mu_{N}=\mu_{0 N} \text { Vs. } H_{1}: \mu_{N} \neq \mu_{O N} .
$$

Where $\mu_{N}$ is a true daily average death and $\mu_{0 N}$ is the specified daily average deaths. Suppose that $t_{0 N}=a \mu_{0 N}$ be the time in days, where $a$ is the termination ratio. The probability of the item will fail before it reaches the experiment time $t_{0 N}$ is defined as follows:

\begin{tabular}{|c|c|c|c|c|c|c|c|c|c|c|c|c|c|c|c|c|c|}
\hline \multirow[t]{2}{*}{$\tilde{\tilde{\beta}}$} & \multirow{2}{*}{$\frac{\mu_{N}}{\mu_{\mathrm{ON}}}$} & \multicolumn{4}{|c|}{$I_{U}=0.00$} & \multicolumn{4}{|c|}{$I_{U}=0.02$} & \multicolumn{4}{|c|}{$I_{U}=0.04$} & \multicolumn{4}{|c|}{$I_{U}=0.05$} \\
\hline & & $\mathrm{n}$ & $c_{1}$ & $c_{2}$ & ASN & $\mathrm{n}$ & $c_{1}$ & $c_{2}$ & ASN & $\mathrm{n}$ & $c_{1}$ & $c_{2}$ & ASN & $n$ & $c_{1}$ & $c_{2}$ & ASN \\
\hline 0.25 & 1.1 & 216 & 126 & 141 & 670.58 & 200 & 120 & 135 & 670.44 & 212 & 133 & 146 & 561.99 & 215 & 137 & 150 & 562.33 \\
\hline 0.25 & 1.2 & 77 & 43 & 50 & 171.96 & 108 & 65 & 70 & 164.07 & 100 & 62 & 67 & 156.47 & 72 & 44 & 50 & 146.39 \\
\hline 0.25 & 1.3 & 40 & 21 & 26 & 87.26 & 46 & 26 & 30 & 78.70 & 43 & 25 & 29 & 75.96 & 54 & 33 & 36 & 75.89 \\
\hline 0.25 & 1.4 & 37 & 20 & 23 & 56.65 & 38 & 21 & 24 & 56.08 & 44 & 26 & 28 & 54.80 & 28 & 16 & 19 & 47.28 \\
\hline 0.25 & 1.5 & 32 & 17 & 19 & 41.59 & 20 & 10 & 13 & 36.88 & 16 & 8 & 11 & 33.79 & 28 & 16 & 18 & 37.25 \\
\hline 0.25 & 1.8 & 16 & 8 & 9 & 19.23 & 13 & 6 & 8 & 20.53 & 11 & 5 & 7 & 18.25 & 18 & 10 & 11 & 21.30 \\
\hline 0.25 & 2.0 & 8 & 3 & 5 & 15.57 & 12 & 5 & 7 & 17.86 & 16 & 9 & 9 & 16.00 & 10 & 5 & 6 & 12.75 \\
\hline 0.10 & 1.1 & - & - & - & - & - & - & - & - & - & - & - & - & - & - & - & - \\
\hline 0.10 & 1.2 & 166 & 95 & 103 & 251.81 & 156 & 92 & 100 & 241.03 & 134 & 81 & 89 & 220.93 & 145 & 90 & 97 & 215.03 \\
\hline 0.10 & 1.3 & 76 & 41 & 47 & 123.27 & 67 & 37 & 43 & 114.90 & 65 & 37 & 43 & 111.98 & 64 & 37 & 43 & 110.92 \\
\hline 0.10 & 1.4 & 49 & 25 & 30 & 79.68 & 51 & 27 & 32 & 79.54 & 36 & 19 & 24 & 69.65 & 42 & 23 & 28 & 72.87 \\
\hline 0.10 & 1.5 & 25 & 11 & 16 & 57.81 & 19 & 8 & 13 & 56.40 & 40 & 22 & 25 & 52.78 & 27 & 14 & 18 & 48.74 \\
\hline 0.10 & 1.8 & 25 & 11 & 14 & 33.38 & 21 & 10 & 12 & 26.67 & 16 & 7 & 10 & 26.17 & 20 & 10 & 12 & 25.48 \\
\hline 0.10 & 2.0 & 21 & 9 & 11 & 25.09 & 13 & 5 & 8 & 23.56 & 9 & 3 & 6 & 20.91 & 19 & 9 & 11 & 23.26 \\
\hline 0.05 & 1.1 & - & - & - & - & - & - & - & - & - & - & - & - & - & - & - & - \\
\hline 0.05 & 1.2 & 180 & 101 & 112 & 294.66 & 158 & 91 & 102 & 278.26 & 147 & 87 & 98 & 266.01 & 150 & 91 & 101 & 251.91 \\
\hline 0.05 & 1.3 & 109 & 59 & 66 & 151.86 & 83 & 45 & 53 & 141.34 & 69 & 38 & 46 & 133.05 & 68 & 38 & 46 & 131.00 \\
\hline 0.05 & 1.4 & 65 & 33 & 39 & 94.37 & 61 & 32 & 38 & 91.56 & 65 & 36 & 41 & 86.23 & 45 & 24 & 30 & 80.22 \\
\hline 0.05 & 1.5 & 30 & 13 & 19 & 67.10 & 42 & 21 & 26 & 64.66 & 34 & 17 & 22 & 57.79 & 37 & 19 & 24 & 58.15 \\
\hline 0.05 & 1.8 & 27 & 12 & 15 & 34.90 & 16 & 6 & 10 & 30.81 & 19 & 8 & 12 & 32.45 & 17 & 7 & 11 & 30.71 \\
\hline 0.05 & 2.0 & 16 & 6 & 9 & 24.06 & 13 & 4 & 8 & 25.03 & 19 & 8 & 11 & 25.01 & 13 & 5 & 8 & 20.73 \\
\hline 0.01 & 1.1 & - & - & - & - & - & - & - & - & - & - & - & - & - & - & - & - \\
\hline 0.01 & 1.2 & 235 & 129 & 146 & 381.10 & 233 & 133 & 149 & 363.60 & 214 & 126 & 141 & 331.60 & 195 & 116 & 131 & 321.50 \\
\hline 0.01 & 1.3 & 110 & 56 & 68 & 186.50 & 119 & 64 & 75 & 178.40 & 114 & 63 & 74 & 168.96 & 91 & 50 & 61 & 160.90 \\
\hline 0.01 & 1.4 & 78 & 38 & 47 & 115.80 & 65 & 32 & 41 & 107.90 & 74 & 39 & 47 & 102.50 & 57 & 29 & 38 & 102.30 \\
\hline 0.01 & 1.5 & 57 & 26 & 34 & 84.89 & 59 & 28 & 36 & 82.77 & 49 & 24 & 31 & 71.92 & 46 & 22 & 30 & 73.98 \\
\hline 0.01 & 1.8 & 31 & 12 & 18 & 46.41 & 31 & 13 & 18 & 41.25 & 28 & 12 & 17 & 39.13 & 24 & 10 & 15 & 36.09 \\
\hline 0.01 & 2.0 & 17 & 5 & 10 & 30.21 & 25 & 9 & 14 & 32.01 & 18 & 6 & 11 & 28.48 & 24 & 10 & 14 & 29.85 \\
\hline
\end{tabular}

$$
\begin{gathered}
p_{N}=1-\left\{e^{-\left(\frac{t_{N} N}{\alpha}\right)^{\beta}}\left(1+I_{N}\right)\right\}+I_{N} ; I_{N} \in\left[I_{L}, I_{U}\right] \\
=1-\left\{\exp \left(-a^{\beta}\left(\mu_{N} / \mu_{0 N}\right)^{-\beta}(\Gamma(1 / \beta) / \beta)^{\beta}\left(1+I_{N}\right)^{\beta}\right)\left(1+I_{N}\right)\right\}+I_{N}
\end{gathered}
$$

Table 2 The plan parameter when $\tilde{a}=0.10 ; \beta=1$ and $a=1.0$ 
where $\mu_{N} / \mu_{0 N}$ is the ratio of true average daily wind speed to specified average daily wind speed. Suppose that $\tilde{\alpha}$ and $\tilde{\beta}$ be type-I and type-II errors. The medical practitioners are interested to apply the proposed plan for testing $H_{0}: \mu_{N}=\mu_{0 N}$ such that the probability of accepting $H_{0}: \mu_{N}=\mu_{0 N}$ when it is true should be larger than $1-\tilde{\alpha}$ at $\mu_{N} / \mu_{0 N}$ and the probability of accepting $H_{0}: \mu_{N}=\mu_{0 N}$ when it is wrong should be smaller than $\tilde{\beta}$ at $\mu_{N} / \mu_{0 N}=1$. In order to find the design parameters $n, c_{1}, c_{2}$ and $I_{N}$ for the proposed RASP, we consider two points on the $\mathrm{OC}$ function. In our approach, the quality level mainly depends on the ratio $\mu_{N} / \mu_{0 N}$. This ratio is helpful for the producer to improve the lot quality. From in producer point of view, the probability of acceptance should be at least $1-\tilde{\alpha}$ at acceptable quality level (AQL), $p_{1 N}$. So, the producer demands the lot should be accepted at various levels of $\mu_{N} / \mu_{0 N}$. Similarly, from in consumer point of view the lot rejection probability should not be exceeded $\tilde{\beta}$ at limiting quality level (LQL), $p_{2 N}$. The design parameters are determined by satisfying the following two inequalities

$$
L\left(p_{1 N} \mid \mu_{N} / \mu_{0 N}\right)=\frac{\sum_{i=0}^{c_{1}}\left(\begin{array}{c}
n \\
i
\end{array}\right) p_{1 N} i\left(1-p_{1 N}\right)^{n-i}}{\sum_{i=0}^{c_{1}}\left(\begin{array}{c}
n \\
i
\end{array}\right) p_{1 N} i\left(1-p_{1 N}\right)^{n-i}+1-\sum_{i=0}^{c_{2}}\left(\begin{array}{c}
n \\
i
\end{array}\right) p_{1 N} i\left(1-p_{1 N}\right)^{n-i}} \geq 1-\tilde{\alpha}
$$

Table 3 The plan parameter when $\tilde{a}=0.10 ; \beta=2$ and $a=0.5$

\begin{tabular}{|c|c|c|c|c|c|c|c|c|c|c|c|c|c|c|c|c|c|}
\hline \multirow[t]{2}{*}{$\tilde{\beta}$} & \multirow[t]{2}{*}{$\frac{\mu_{N}}{\mu_{0 N}}$} & \multicolumn{4}{|c|}{$I_{U}=0.00$} & \multicolumn{4}{|c|}{$I_{U}=0.02$} & \multicolumn{4}{|c|}{$I_{U}=0.04$} & \multicolumn{4}{|c|}{$I_{U}=0.05$} \\
\hline & & $\mathrm{n}$ & $c_{1}$ & $c_{2}$ & ASN & $\bar{n}$ & $c_{1}$ & $c_{2}$ & ASN & $\bar{n}$ & $c_{1}$ & $c_{2}$ & ASN & $\mathrm{n}$ & $c_{1}$ & $c_{2}$ & ASN \\
\hline 0.25 & 1.1 & 256 & 38 & 47 & 517.20 & 284 & 46 & 54 & 491.58 & 293 & 51 & 58 & 456.12 & 319 & 58 & 64 & 449.42 \\
\hline 0.25 & 1.2 & 98 & 13 & 17 & 155.09 & 71 & 9 & 14 & 150.09 & 62 & 8 & 13 & 136.88 & 60 & 8 & 13 & 134.22 \\
\hline 0.25 & 1.3 & 53 & 6 & 9 & 85.43 & 51 & 6 & 9 & 80.86 & 48 & 6 & 9 & 76.77 & 26 & 2 & 6 & 74.76 \\
\hline 0.25 & 1.4 & 38 & 4 & 6 & 54.61 & 37 & 4 & 6 & 52.16 & 28 & 3 & 5 & 42.59 & 33 & 4 & 6 & 47.73 \\
\hline 0.25 & 1.5 & 25 & 2 & 4 & 40.34 & 31 & 3 & 5 & 45.48 & 33 & 4 & 5 & 38.75 & 22 & 2 & 4 & 35.43 \\
\hline 0.25 & 1.8 & 28 & 3 & 3 & 28.00 & 16 & 1 & 2 & 20.75 & 15 & 1 & 2 & 19.54 & 14 & 1 & 2 & 18.53 \\
\hline 0.25 & 2.0 & 21 & 2 & 2 & 21.00 & 21 & 2 & 2 & 21.00 & 20 & 2 & 2 & 20.00 & 8 & 0 & 1 & 11.94 \\
\hline 0.10 & 1.1 & 462 & 69 & 81 & 778.83 & 425 & 67 & 79 & 731.36 & 345 & 56 & 69 & 693.87 & 421 & 73 & 84 & 666.03 \\
\hline 0.10 & 1.2 & 149 & 19 & 25 & 231.97 & 113 & 14 & 21 & 218.82 & 127 & 18 & 24 & 204.39 & 92 & 12 & 19 & 197.78 \\
\hline 0.10 & 1.3 & 72 & 7 & 12 & 126.10 & 77 & 9 & 13 & 114.70 & 73 & 9 & 13 & 108.58 & 71 & 9 & 13 & 105.81 \\
\hline 0.10 & 1.4 & 62 & 6 & 9 & 83.19 & 58 & 6 & 9 & 78.82 & 48 & 5 & 8 & 68.56 & 37 & 3 & 7 & 69.39 \\
\hline 0.10 & 1.5 & 42 & 3 & 6 & 61.36 & 31 & 2 & 5 & 52.13 & 29 & 2 & 5 & 49.61 & 25 & 1 & 5 & 58.24 \\
\hline 0.10 & 1.8 & 23 & 1 & 3 & 34.22 & 22 & 1 & 3 & 32.40 & 18 & 0 & 3 & 35.08 & 20 & 1 & 3 & 29.81 \\
\hline 0.10 & 2.0 & 15 & 0 & 2 & 26.36 & 14 & 0 & 2 & 24.93 & 15 & 0 & 2 & 23.66 & 13 & 0 & 2 & 22.99 \\
\hline 0.05 & 1.1 & 486 & 70 & 86 & 921.90 & 559 & 88 & 102 & 860.99 & 518 & 86 & 100 & 809.80 & 453 & 76 & 91 & 789.07 \\
\hline 0.05 & 1.2 & 173 & 21 & 29 & 276.86 & 157 & 20 & 28 & 258.41 & 142 & 19 & 27 & 243.33 & 145 & 20 & 28 & 237.39 \\
\hline 0.05 & 1.3 & 93 & 9 & 15 & 148.49 & 105 & 12 & 17 & 142.08 & 69 & 7 & 13 & 127.94 & 90 & 11 & 16 & 126.23 \\
\hline 0.05 & 1.4 & 56 & 4 & 9 & 97.89 & 45 & 3 & 8 & 89.79 & 58 & 5 & 10 & 90.71 & 59 & 6 & 10 & 82.73 \\
\hline 0.05 & 1.5 & 46 & 3 & 7 & 74.60 & 36 & 2 & 6 & 64.99 & 42 & 3 & 7 & 65.87 & 33 & 2 & 6 & 60.20 \\
\hline 0.05 & 1.8 & 44 & 3 & 5 & 51.50 & 19 & 0 & 3 & 37.07 & 18 & 0 & 3 & 35.08 & 17 & 0 & 3 & 34.70 \\
\hline 0.05 & 2.0 & 28 & 1 & 3 & 35.44 & 26 & 1 & 3 & 33.32 & 24 & 1 & 3 & 31.33 & 23 & 1 & 3 & 30.38 \\
\hline 0.01 & 1.1 & - & - & - & - & - & - & - & - & - & - & - & - & - & - & - & - \\
\hline 0.01 & 1.2 & 206 & 23 & 35 & 351.10 & 195 & 23 & 35 & 331.10 & 219 & 29 & 40 & 312.40 & 207 & 28 & 39 & 301.03 \\
\hline 0.01 & 1.3 & 135 & 13 & 21 & 187.90 & 113 & 11 & 19 & 169.80 & 113 & 12 & 20 & 166.28 & 97 & 10 & 18 & 154.38 \\
\hline 0.01 & 1.4 & 88 & 7 & 13 & 120.60 & 84 & 7 & 13 & 113.10 & 79 & 7 & 13 & 107.50 & 77 & 7 & 13 & 104.49 \\
\hline 0.01 & 1.5 & 64 & 4 & 9 & 87.32 & 60 & 4 & 9 & 82.88 & 54 & 3 & 9 & 82.24 & 56 & 4 & 9 & 75.69 \\
\hline 0.01 & 1.8 & 27 & 0 & 4 & 49.47 & 38 & 1 & 5 & 50.64 & 35 & 1 & 5 & 48.14 & 39 & 2 & 5 & 46.16 \\
\hline 0.01 & 2.0 & 40 & 1 & 4 & 46.08 & 35 & 1 & 4 & 42.60 & 32 & 1 & 4 & 40.05 & 22 & 0 & 3 & 31.76 \\
\hline
\end{tabular}




$$
L\left(p_{2 N} \mid \mu_{N} / \mu_{0 N}=1\right)=\frac{\sum_{i=0}^{c_{1}}\left(\begin{array}{c}
n \\
i
\end{array}\right) p_{2 N}{ }^{i}\left(1-p_{2 N}\right)^{n-i}}{\sum_{i=0}^{c_{1}}\left(\begin{array}{c}
n \\
i
\end{array}\right) p_{2 N} i\left(1-p_{2 N}\right)^{n-i}+1-\sum_{i=0}^{c_{2}}\left(\begin{array}{c}
n \\
i
\end{array}\right) p_{2 N} i\left(1-p_{2 N}\right)^{n-i}} \leq \tilde{\beta}
$$

where $p_{1 N}$ and $p_{2 N}$ are defined by

$$
\begin{aligned}
& p_{1 N}=1-\left\{\exp \left(-a^{\beta}\left(\mu / \mu_{0}\right)^{-\beta}(\Gamma(1 / \beta) / \beta)^{\beta}\left(1+I_{N}\right)^{\beta}\right)\left(1+I_{N}\right)\right\}+I_{N} \\
& p_{2 N}=1-\left\{\exp \left(-a^{\beta}(\Gamma(1 / \beta) / \beta)^{\beta}\left(1+I_{N}\right)^{\beta}\right)\left(1+I_{N}\right)\right\}+I_{N}
\end{aligned}
$$

The estimated designed parameters of the proposed plan should be minimizing the average sample number (ASN) at an acceptable quality level. The ASN for the proposed plan with fraction defective $(p)$ is derived to be

$$
A S N=\frac{n}{P_{a}(p)+P_{r}(p)}
$$

Therefore, the design parameters for the proposed plan with minimum sample size will be obtained by solving the below optimization technique

$$
\begin{gathered}
\text { Minimize } A S N\left(p_{1 N}\right) \\
\text { subject to } \\
L\left(p_{1 N}\right) \geq 1-\tilde{\alpha} \\
L\left(p_{2 N}\right) \leq \tilde{\beta} \\
0 \leq c_{1} \leq c_{2}
\end{gathered}
$$

\begin{tabular}{|c|c|c|c|c|c|c|c|c|c|c|c|c|c|c|c|c|c|}
\hline \multirow[t]{2}{*}{$\tilde{\beta}$} & \multirow[t]{2}{*}{$\frac{\mu_{N}}{\mu_{0 N}}$} & \multicolumn{4}{|c|}{$I_{U}=0.00$} & \multicolumn{4}{|c|}{$I_{U}=0.02$} & \multicolumn{4}{|c|}{$I_{U}=0.04$} & \multicolumn{4}{|c|}{$I_{U}=0.05$} \\
\hline & & $n$ & $c_{1}$ & $c_{2}$ & ASN & $n$ & $c_{1}$ & $c_{2}$ & ASN & $n$ & $c_{1}$ & $c_{2}$ & ASN & $n$ & $c_{1}$ & $c_{2}$ & ASN \\
\hline 0.255 & 1.1 & 139 & 70 & 74 & 180.96 & 98 & 50 & 56 & 167.43 & 105 & 57 & 62 & 158.45 & 83 & 45 & 51 & 152.44 \\
\hline 0.25 & 1.2 & 27 & 11 & 15 & 55.27 & 39 & 19 & 21 & 49.78 & 31 & 15 & 18 & 48.58 & 30 & 15 & 18 & 48.38 \\
\hline 0.25 & 1.3 & 17 & 6 & 9 & 31.20 & 19 & 8 & 10 & 26.98 & 18 & 8 & 10 & 26.05 & 19 & 9 & 11 & 27.77 \\
\hline 0.25 & 1.4 & 17 & 7 & 8 & 20.21 & 11 & 4 & 6 & 18.62 & 11 & 4 & 6 & 17.60 & 18 & 9 & 9 & 18.00 \\
\hline 0.25 & 1.5 & 11 & 4 & 5 & 13.71 & 19 & 9 & 8 & 16.85 & 8 & 3 & 4 & 10.47 & 5 & 1 & 3 & 11.57 \\
\hline 0.25 & 1.8 & 5 & 1 & 2 & 6.95 & 8 & 3 & 3 & 8.00 & 3 & 0 & 2 & 10.82 & 6 & 2 & 3 & 8.23 \\
\hline 0.25 & 2.0 & 6 & 1 & 2 & 7.42 & 5 & 1 & 2 & 6.75 & 6 & 2 & 2 & 6.00 & 9 & 4 & 3 & 7.76 \\
\hline 0.10 & 1.1 & 138 & 65 & 75 & 263.63 & 169 & 86 & 94 & 250.98 & 134 & 70 & 79 & 233.82 & 137 & 74 & 82 & 220.09 \\
\hline 0.10 & 1.2 & 57 & 25 & 29 & 78.21 & 41 & 17 & 23 & 79.30 & 45 & 21 & 26 & 75.79 & 39 & 18 & 23 & 67.40 \\
\hline 0.10 & 1.3 & 25 & 9 & 13 & 43.98 & 25 & 10 & 13 & 36.43 & 24 & 10 & 13 & 34.72 & 30 & 14 & 16 & 36.26 \\
\hline 0.10 & 1.4 & 16 & 5 & 8 & 26.78 & 15 & 5 & 8 & 26.29 & 22 & 9 & 11 & 26.89 & 12 & 4 & 7 & 23.41 \\
\hline 0.10 & 1.5 & 10 & 2 & 5 & 19.27 & 15 & 5 & 7 & 19.59 & 11 & 3 & 6 & 19.95 & 11 & 3 & 6 & 18.92 \\
\hline 0.10 & 1.8 & 9 & 2 & 4 & 13.62 & 4 & 0 & 2 & 8.74 & 10 & 3 & 4 & 11.31 & 10 & 3 & 4 & 11.16 \\
\hline 0.10 & 2.0 & 9 & 2 & 3 & 10.25 & 4 & 0 & 2 & 8.74 & 8 & 1 & 3 & 9.66 & 8 & 2 & 3 & 9.05 \\
\hline 0.05 & 1.1 & 189 & 89 & 101 & 313.09 & 181 & 90 & 101 & 289.79 & 181 & 95 & 105 & 271.36 & 170 & 91 & 101 & 261.00 \\
\hline 0.05 & 1.2 & 56 & 23 & 29 & 88.23 & 59 & 26 & 32 & 91.16 & 49 & 22 & 28 & 80.65 & 46 & 21 & 27 & 78.19 \\
\hline 0.05 & 1.3 & 37 & 14 & 18 & 50.18 & 31 & 12 & 16 & 44.56 & 32 & 13 & 17 & 43.38 & 25 & 10 & 14 & 38.63 \\
\hline 0.05 & 1.4 & 26 & 9 & 12 & 33.51 & 17 & 5 & 9 & 30.35 & 12 & 3 & 7 & 27.18 & 21 & 8 & 11 & 28.07 \\
\hline 0.05 & 1.5 & 19 & 6 & 8 & 22.62 & 12 & 3 & 6 & 19.74 & 14 & 4 & 7 & 20.04 & 11 & 3 & 6 & 18.92 \\
\hline 0.05 & 1.8 & 10 & 2 & 4 & 13.24 & 10 & 2 & 4 & 12.52 & 8 & 1 & 4 & 13.51 & 9 & 2 & 4 & 11.65 \\
\hline 0.05 & 2.0 & 7 & 0 & 3 & 11.67 & 9 & 2 & 3 & 9.99 & 8 & 1 & 3 & 9.66 & 9 & 2 & 4 & 11.65 \\
\hline 0.01 & 1.1 & 272 & 127 & 144 & 405.20 & 255 & 125 & 141 & 370.60 & 229 & 117 & 133 & 349.70 & 184 & 94 & 111 & 345.60 \\
\hline 0.01 & 1.2 & 78 & 31 & 40 & 115.10 & 72 & 30 & 39 & 111.20 & 70 & 31 & 39 & 98.57 & 56 & 24 & 33 & 98.24 \\
\hline 0.01 & 1.3 & 35 & 11 & 18 & 60.36 & 39 & 14 & 20 & 54.33 & 37 & 14 & 20 & 52.56 & 32 & 12 & 18 & 49.45 \\
\hline 0.01 & 1.4 & 33 & 10 & 15 & 40.78 & 29 & 9 & 14 & 37.17 & 25 & 8 & 13 & 34.68 & 27 & 9 & 14 & 34.57 \\
\hline 0.01 & 1.5 & 19 & 4 & 9 & 29.01 & 28 & 9 & 12 & 30.69 & 18 & 5 & 9 & 24.68 & 17 & 4 & 9 & 25.42 \\
\hline 0.01 & 1.8 & 13 & 2 & 5 & 15.97 & 9 & 1 & 4 & 13.37 & 12 & 2 & 5 & 14.35 & 11 & 2 & 5 & 14.17 \\
\hline 0.01 & 2.0 & 15 & 3 & 5 & 16.25 & 12 & 2 & 4 & 13.07 & 6 & 0 & 3 & 11.13 & 6 & 0 & 3 & 10.62 \\
\hline
\end{tabular}

where $n, c_{1}, c_{2} \in z$

The values of the designed parameters $n, c_{1}$ and $c_{2}$ for various values of $\tilde{\beta}=0.25,0.10,0.05,0.01 ; \tilde{\alpha}=0.10$; $a=$ 0.5 and $1.0, \mu_{N} / \mu_{0 N}=1.1,1.2,1.3,1.4,1.5,1.8,2.0$ and $I_{N}$ $=0.0,0.02,0.04$ and 0.05 when shape parameter $\beta=1,2$

Table 4 The plan parameter when $\tilde{a}=0.10 ; \beta=2$ and $a=1.00$ 
and 3 are given in Tables $1,2,3,4,5$ and 6 . Tables 1 and 2 are shown for the exponential distribution case. For exponential distribution, it can be seen that the values of ASN decrease as the values of $a$ increases from 0.5 to 1.0. On the other hand for other the same parameters, the values of $n$ decreases as the values of $\beta$ increases. Note here that the indeterminacy parameter $I_{N}$ also plays a significant role in minimizing the sample size. As indeterminacy parameter $I_{N}$ increases the ASN values are decreasing.

\section{Results}

A comparative study is carried out between the proposed sampling plans with the existing sampling plans available in the literature with respect to the sample size in this section. We know the cost of the study is always directly proportional to the sample size, a plan is said to be economical if it requires a smaller number of samples for testing the hypothesis about the daily new deaths from COVID-19. The proposed repetitive sampling plan under uncertainty/indeterminacy for Weibull distribution is the generalization of the testing average wind speed using sampling plan for Weibull distribution under indeterminacy plan developed by [42]. The comparison for the proposed and the existing sampling plan for Weibull distribution under indeterminacy plan developed by [42] are displayed in Tables 7 and 8 for $\tilde{\alpha}=$ $0.10 ; \beta=2$ at $a=0.5$ and 1.0. The developed sampling plan reduces to the existing sampling plan when $c_{1}=$ $c_{2}=c$. From Tables 7 and 8 , it is noticed that the values of the sample size required for testing $H_{0}: \mu_{N}=\mu_{0 N}$ smaller for the proposed sampling plan as compared

Table 5 The plan parameter when $\tilde{a}=0.10 ; \beta=3$ and $a=0.5$

\begin{tabular}{|c|c|c|c|c|c|c|c|c|c|c|c|c|c|c|c|c|c|}
\hline \multirow[t]{2}{*}{$\tilde{\beta}$} & \multirow[t]{2}{*}{$\frac{\mu_{N}}{\mu_{O N}}$} & \multicolumn{4}{|c|}{$I_{U}=0.00$} & \multicolumn{4}{|c|}{$I_{U}=0.02$} & \multicolumn{4}{|c|}{$I_{U}=0.04$} & \multicolumn{4}{|c|}{$I_{U}=0.05$} \\
\hline & & $\bar{n}$ & $c_{1}$ & $c_{2}$ & ASN & $\mathrm{n}$ & $c_{1}$ & $c_{2}$ & ASN & $\bar{n}$ & $c_{1}$ & $c_{2}$ & ASN & $\mathrm{n}$ & $c_{1}$ & $c_{2}$ & ASN \\
\hline 0.25 & 1.1 & 292 & 19 & 25 & 517.61 & 245 & 17 & 23 & 458.13 & 277 & 22 & 27 & 429.66 & 267 & 22 & 27 & 414.40 \\
\hline 0.25 & 1.2 & 85 & 4 & 7 & 146.81 & 91 & 5 & 8 & 149.65 & 73 & 4 & 7 & 126.55 & 70 & 4 & 7 & 122.04 \\
\hline 0.25 & 1.3 & 30 & 0 & 3 & 94.07 & 51 & 2 & 4 & 78.45 & 46 & 2 & 4 & 72.19 & 44 & 2 & 4 & 69.43 \\
\hline 0.25 & 1.4 & 25 & 0 & 2 & 53.46 & 46 & 2 & 3 & 56.55 & 42 & 2 & 3 & 51.90 & 21 & 0 & 2 & 44.60 \\
\hline 0.25 & 1.5 & 35 & 1 & 2 & 45.38 & 35 & 1 & 2 & 44.23 & 30 & 1 & 2 & 38.96 & 30 & 1 & 2 & 38.48 \\
\hline 0.25 & 1.8 & 20 & 0 & 1 & 29.15 & 21 & 0 & 1 & 29.19 & 17 & 0 & 1 & 24.90 & 27 & 1 & 1 & 27.00 \\
\hline 0.25 & 2.0 & 20 & 0 & 1 & 29.15 & 20 & 0 & 1 & 28.34 & 18 & 0 & 1 & 25.81 & 17 & 0 & 1 & 24.57 \\
\hline 0.10 & 1.1 & 403 & 25 & 34 & 759.91 & 443 & 31 & 39 & 701.00 & 399 & 30 & 38 & 642.41 & 385 & 30 & 38 & 617.97 \\
\hline 0.10 & 1.2 & 123 & 5 & 10 & 235.19 & 149 & 8 & 12 & 218.34 & 138 & 8 & 12 & 202.77 & 133 & 8 & 12 & 195.45 \\
\hline 0.10 & 1.3 & 111 & 5 & 7 & 135.49 & 55 & 1 & 5 & 128.82 & 74 & 3 & 6 & 111.25 & 50 & 1 & 5 & 113.77 \\
\hline 0.10 & 1.4 & 38 & 0 & 3 & 86.02 & 34 & 0 & 3 & 80.99 & 58 & 2 & 4 & 76.59 & 56 & 2 & 4 & 73.83 \\
\hline 0.10 & 1.5 & 63 & 2 & 3 & 71.39 & 47 & 1 & 3 & 67.15 & 45 & 1 & 3 & 62.66 & 41 & 1 & 3 & 59.74 \\
\hline 0.10 & 1.8 & 47 & 1 & 2 & 54.83 & 43 & 1 & 2 & 50.37 & 41 & 1 & 2 & 47.54 & 27 & 0 & 2 & 45.89 \\
\hline 0.10 & 2.0 & 32 & 0 & 1 & 38.68 & 31 & 0 & 1 & 36.81 & 28 & 0 & 1 & 33.57 & 24 & 0 & 1 & 30.13 \\
\hline 0.05 & 1.1 & 494 & 30 & 41 & 890.71 & 470 & 31 & 42 & 834.52 & 412 & 29 & 40 & 764.15 & 432 & 32 & 43 & 751.53 \\
\hline 0.05 & 1.2 & 190 & 9 & 14 & 274.71 & 139 & 6 & 12 & 257.00 & 128 & 6 & 12 & 241.07 & 157 & 9 & 14 & 227.78 \\
\hline 0.05 & 1.3 & 100 & 3 & 7 & 153.78 & 110 & 4 & 8 & 154.20 & 69 & 2 & 6 & 123.22 & 82 & 3 & 7 & 127.89 \\
\hline 0.05 & 1.4 & 61 & 1 & 4 & 96.63 & 56 & 1 & 4 & 89.64 & 52 & 1 & 4 & 83.11 & 70 & 2 & 5 & 92.28 \\
\hline 0.05 & 1.5 & 44 & 0 & 3 & 81.11 & 43 & 0 & 3 & 73.85 & 37 & 0 & 3 & 70.19 & 38 & 0 & 3 & 66.19 \\
\hline 0.05 & 1.8 & 41 & 0 & 2 & 57.27 & 36 & 0 & 2 & 52.46 & 34 & 0 & 2 & 48.78 & 33 & 0 & 2 & 47.05 \\
\hline 0.05 & 2.0 & 36 & 0 & 1 & 41.67 & 34 & 0 & 1 & 39.07 & 32 & 0 & 1 & 36.56 & 30 & 0 & 1 & 34.59 \\
\hline 0.01 & 1.1 & - & - & - & - & - & - & - & - & - & - & - & - & - & - & - & - \\
\hline 0.01 & 1.2 & 241 & 10 & 18 & 356.05 & 223 & 10 & 18 & 330.00 & 222 & 11 & 19 & 312.30 & 185 & 9 & 17 & 289.40 \\
\hline 0.01 & 1.3 & 155 & 5 & 10 & 197.70 & 116 & 3 & 9 & 182.40 & 133 & 5 & 10 & 169.60 & 104 & 3 & 9 & 162.40 \\
\hline 0.01 & 1.4 & 100 & 2 & 6 & 130.80 & 92 & 2 & 6 & 121.10 & 74 & 1 & 6 & 120.80 & 82 & 2 & 6 & 108.20 \\
\hline 0.01 & 1.5 & 61 & 0 & 4 & 100.60 & 56 & 0 & 4 & 93.47 & 52 & 0 & 4 & 86.59 & 50 & 0 & 4 & 83.51 \\
\hline 0.01 & 1.8 & 54 & 0 & 2 & 62.97 & 53 & 0 & 3 & 72.01 & 51 & 0 & 3 & 66.94 & 46 & 0 & 3 & 64.22 \\
\hline 0.01 & 2.0 & 54 & 0 & 2 & 62.97 & 52 & 0 & 2 & 59.44 & 49 & 0 & 2 & 55.56 & 45 & 0 & 2 & 52.19 \\
\hline
\end{tabular}


Table 6 The plan parameter when $\tilde{a}=0.10 ; \beta=3$ and $a=1.0$

\begin{tabular}{|c|c|c|c|c|c|c|c|c|c|c|c|c|c|c|c|c|c|}
\hline \multirow[t]{2}{*}{$\tilde{\beta}$} & \multirow{2}{*}{$\frac{\mu_{N}}{\mu_{\mathrm{ON}}}$} & \multicolumn{4}{|c|}{$I_{U}=0.00$} & \multicolumn{4}{|c|}{$I_{U}=0.02$} & \multicolumn{4}{|c|}{$I_{U}=0.04$} & \multicolumn{4}{|c|}{$I_{U}=0.05$} \\
\hline & & $\mathrm{n}$ & $c_{1}$ & $c_{2}$ & ASN & $\mathrm{n}$ & $c_{1}$ & $c_{2}$ & ASN & $\mathbf{n}$ & $c_{1}$ & $c_{2}$ & ASN & $\mathrm{n}$ & $c_{1}$ & $c_{2}$ & ASN \\
\hline 0.25 & 1.1 & 78 & 32 & 39 & 136.44 & 65 & 31 & 34 & 86.96 & 66 & 34 & 36 & 79.07 & 46 & 23 & 27 & 77.00 \\
\hline 0.25 & 1.2 & 25 & 8 & 12 & 42.95 & 23 & 10 & 11 & 26.67 & 17 & 7 & 9 & 24.71 & 15 & 6 & 9 & 31.15 \\
\hline 0.25 & 1.3 & 19 & 6 & 8 & 24.45 & 11 & 4 & 5 & 13.76 & 10 & 3 & 5 & 15.57 & 5 & 1 & 3 & 12.06 \\
\hline 0.25 & 1.4 & 13 & 3 & 5 & 16.83 & 9 & 2 & 4 & 13.73 & 10 & 4 & 4 & 10.00 & 8 & 2 & 4 & 12.77 \\
\hline 0.25 & 1.5 & 11 & 2 & 4 & 14.20 & 9 & 3 & 3 & 9.00 & 8 & 3 & 3 & 8.00 & 7 & 2 & 3 & 8.79 \\
\hline 0.25 & 1.8 & 4 & 0 & 1 & 5.27 & 3 & 0 & 1 & 4.56 & 4 & 1 & 1 & 4.00 & 4 & 1 & 1 & 4.00 \\
\hline 0.25 & 2.0 & 4 & 0 & 1 & 5.27 & 3 & 0 & 1 & 4.56 & 4 & 1 & 1 & 4.00 & 4 & 1 & 1 & 4.00 \\
\hline 0.10 & 1.1 & 44 & 18 & 23 & 89.62 & 59 & 25 & 32 & 120.98 & 68 & 32 & 38 & 112.30 & 64 & 31 & 37 & 109.60 \\
\hline 0.10 & 1.2 & 16 & 5 & 8 & 30.57 & 27 & 10 & 13 & 37.35 & 20 & 7 & 11 & 37.37 & 15 & 5 & 9 & 36.41 \\
\hline 0.10 & 1.3 & 11 & 3 & 5 & 17.54 & 15 & 4 & 7 & 22.85 & 17 & 6 & 8 & 21.59 & 9 & 2 & 5 & 18.53 \\
\hline 0.10 & 1.4 & 10 & 3 & 4 & 12.46 & 13 & 4 & 5 & 14.73 & 9 & 2 & 4 & 12.65 & 14 & 5 & 6 & 15.58 \\
\hline 0.10 & 1.5 & 9 & 2 & 3 & 10.65 & 7 & 1 & 3 & 11.14 & 8 & 2 & 3 & 9.41 & 4 & 0 & 2 & 8.24 \\
\hline 0.10 & 1.8 & 3 & 0 & 1 & 4.75 & 8 & 2 & 2 & 8.00 & 6 & 1 & 2 & 7.17 & 3 & 0 & 1 & 4.27 \\
\hline 0.10 & 2.0 & 3 & 0 & 1 & 4.75 & 7 & 0 & 2 & 8.34 & 4 & 0 & 1 & 4.87 & 4 & 0 & 1 & 4.78 \\
\hline 0.05 & 1.1 & 98 & 40 & 48 & 152.82 & 95 & 42 & 49 & 138.53 & 69 & 31 & 39 & 129.53 & 81 & 39 & 46 & 125.46 \\
\hline 0.05 & 1.2 & 22 & 6 & 11 & 46.59 & 28 & 9 & 14 & 45.58 & 27 & 10 & 14 & 39.85 & 19 & 6 & 11 & 40.85 \\
\hline 0.05 & 1.3 & 16 & 4 & 7 & 24.25 & 18 & 5 & 8 & 24.21 & 14 & 4 & 7 & 21.72 & 14 & 4 & 7 & 20.45 \\
\hline 0.05 & 1.4 & 17 & 4 & 6 & 19.47 & 15 & 4 & 6 & 18.10 & 8 & 1 & 4 & 14.70 & 10 & 2 & 5 & 16.09 \\
\hline 0.05 & 1.5 & 8 & 1 & 3 & 11.62 & 12 & 3 & 4 & 13.10 & 11 & 3 & 4 & 12.11 & 7 & 1 & 3 & 9.84 \\
\hline 0.05 & 1.8 & 6 & 0 & 2 & 8.73 & 7 & 1 & 2 & 8.00 & 6 & 0 & 2 & 7.62 & 5 & 0 & 2 & 7.40 \\
\hline 0.05 & 2.0 & 5 & 0 & 1 & 5.87 & 6 & 0 & 2 & 8.12 & 7 & 1 & 2 & 7.76 & 4 & 0 & 1 & 4.78 \\
\hline 0.01 & 1.1 & 136 & 54 & 66 & 197.50 & 113 & 47 & 59 & 180.40 & 107 & 48 & 59 & 165.30 & 104 & 48 & 59 & 160.40 \\
\hline 0.01 & 1.2 & 33 & 9 & 16 & 60.20 & 37 & 12 & 18 & 52.96 & 34 & 11 & 18 & 53.17 & 36 & 13 & 19 & 49.51 \\
\hline 0.01 & 1.3 & 23 & 5 & 10 & 32.96 & 16 & 3 & 8 & 29.75 & 24 & 7 & 11 & 28.94 & 17 & 4 & 9 & 27.88 \\
\hline 0.01 & 1.4 & 11 & 1 & 5 & 20.74 & 18 & 4 & 7 & 20.91 & 17 & 4 & 7 & 19.56 & 14 & 2 & 7 & 21.09 \\
\hline 0.01 & 1.5 & 12 & 1 & 5 & 18.73 & 10 & 1 & 4 & 13.81 & 13 & 2 & 5 & 15.03 & 9 & 1 & 4 & 12.57 \\
\hline 0.01 & 1.8 & 7 & 0 & 2 & 8.80 & 10 & 1 & 3 & 11.21 & 6 & 0 & 2 & 7.62 & 8 & 0 & 3 & 9.87 \\
\hline 0.01 & 2.0 & 8 & 0 & 2 & 9.19 & 8 & 0 & 2 & 8.84 & 8 & 0 & 2 & 8.58 & 7 & 0 & 2 & 7.82 \\
\hline
\end{tabular}

with the existing sampling plan developed by [42]. For example, when $\mu_{N} / \mu_{0 N}=1.1$ and $a=0.5$ from Table 7 , it can be seen that $\mathrm{ASN}=491.58$ from the plan proposed sampling plan whereas existing sampling plan sample size $n=617$ when $I_{N}=0.02, \beta=2$ and $a=0.5$. Hence, the proposed sampling plan is more economical than the existing sampling plan.

\section{Discussions}

At this juncture, application of the proposed methodology will be illustrated using COVID-19 data belong to Italy of 111 days that are recorded from 1 April to 20 July 2020. The data are available at https://covid19.who. int/. This data is made up of the ratio of daily new deaths (i.e. daily number of deaths over new cases). The data is reported in Table 9. We have taken this data from [46] and they studied applications of COVID-19 data for Kumaraswamy inverted Topp-Leone distribution. Coronavirus disease (COVID-19) is an infectious disease caused by a newly discovered coronavirus. A large number of people affected by the COVID-19 virus and it are infected at random and uncertain, the COVID-19 data follows a certain statistical distribution under neutrosophic statistics. The World health organization and different countries' health administrators are involved to check the daily affected cases, recovered cases and deaths under indeterminacy. It is found that the COVID-19 data follows the Weibull distribution with shape parameter $\hat{\beta}=2.2222$ with the standard error (SE) as 0.1596 and scale parameter $\hat{\alpha}=0.1880$ with $\mathrm{SE}$ value as 0.00845 . The Kolmogorov-Smirnov test and it $p$ value are $\mathrm{D}=0.0684$ and $p=0.6766$. The goodness of fit of the Weibull distribution is highlight by 
Table 7 Sample size comparison between the proposed plan and existing plan for $\tilde{a}=0.10 ; \beta=2$ and $a=0.5$

\begin{tabular}{|c|c|c|c|c|c|c|c|c|c|}
\hline \multirow[t]{2}{*}{$\tilde{\beta}$} & \multirow[t]{2}{*}{$\frac{\mu_{N}}{\mu_{0 N}}$} & \multicolumn{2}{|l|}{$I_{U}=0.00$} & \multicolumn{2}{|l|}{$I_{U}=0.02$} & \multicolumn{2}{|l|}{$I_{U}=0.04$} & \multicolumn{2}{|l|}{$I_{U}=0.05$} \\
\hline & & Proposed & Existing & Proposed & Existing & Proposed & Existing & Proposed & Existing \\
\hline 0.25 & 1.1 & 517.20 & 646 & 491.58 & 617 & 456.12 & 573 & 449.42 & 558 \\
\hline 0.25 & 1.2 & 155.09 & 198 & 150.09 & 181 & 136.88 & 172 & 134.22 & 167 \\
\hline 0.25 & 1.3 & 85.43 & 110 & 80.86 & 103 & 76.77 & 97 & 74.76 & 94 \\
\hline 0.25 & 1.4 & 54.61 & 66 & 52.16 & 62 & 42.59 & 59 & 47.73 & 58 \\
\hline 0.25 & 1.5 & 40.34 & 47 & 45.48 & 45 & 38.75 & 42 & 35.43 & 41 \\
\hline 0.25 & 1.8 & 28.00 & 29 & 20.75 & 27 & 19.54 & 25 & 18.53 & 25 \\
\hline 0.25 & 2.0 & 21.00 & 21 & 21.00 & 20 & 20.00 & 19 & 11.94 & 19 \\
\hline 0.10 & 1.1 & 778.83 & 1122 & 731.36 & 1049 & 693.87 & 993 & 666.03 & 967 \\
\hline 0.10 & 1.2 & 231.97 & 327 & 218.82 & 315 & 204.39 & 298 & 197.78 & 285 \\
\hline 0.10 & 1.3 & 126.10 & 174 & 114.70 & 164 & 108.58 & 155 & 105.81 & 151 \\
\hline 0.10 & 1.4 & 83.19 & 117 & 78.82 & 110 & 68.56 & 105 & 69.39 & 101 \\
\hline 0.10 & 1.5 & 61.36 & 84 & 52.13 & 79 & 49.61 & 76 & 58.24 & 73 \\
\hline 0.10 & 1.8 & 34.22 & 50 & 32.40 & 47 & 35.08 & 45 & 29.81 & 44 \\
\hline 0.10 & 2.0 & 26.36 & 36 & 24.93 & 34 & 23.66 & 32 & 22.99 & 31 \\
\hline 0.05 & 1.1 & 921.90 & 1467 & 860.99 & 1370 & 809.80 & 1297 & 789.07 & 1257 \\
\hline 0.05 & 1.2 & 276.86 & 435 & 258.41 & 411 & 243.33 & 383 & 237.39 & 373 \\
\hline 0.05 & 1.3 & 148.49 & 230 & 142.08 & 218 & 127.94 & 213 & 126.23 & 200 \\
\hline 0.05 & 1.4 & 97.89 & 153 & 89.79 & 145 & 90.71 & 142 & 82.73 & 132 \\
\hline 0.05 & 1.5 & 74.60 & 112 & 64.99 & 106 & 65.87 & 100 & 60.20 & 98 \\
\hline 0.05 & 1.8 & 51.50 & 64 & 37.07 & 60 & 35.08 & 57 & 34.70 & 55 \\
\hline 0.05 & 2.0 & 35.44 & 49 & 33.32 & 46 & 31.33 & 44 & 30.38 & 44 \\
\hline 0.01 & 1.1 & - & 646 & - & 617 & - & 573 & - & 558 \\
\hline 0.01 & 1.2 & 351.10 & 198 & 331.10 & 181 & 312.40 & 172 & 301.03 & 167 \\
\hline 0.01 & 1.3 & 187.90 & 110 & 169.80 & 103 & 166.28 & 97 & 154.38 & 94 \\
\hline 0.01 & 1.4 & 120.60 & 66 & 113.10 & 62 & 107.50 & 59 & 104.49 & 58 \\
\hline 0.01 & 1.5 & 87.32 & 47 & 82.88 & 45 & 82.24 & 42 & 75.69 & 41 \\
\hline 0.01 & 1.8 & 49.47 & 29 & 50.64 & 27 & 48.14 & 25 & 46.16 & 25 \\
\hline 0.01 & 2.0 & 46.08 & 21 & 42.60 & 20 & 40.05 & 19 & 31.76 & 19 \\
\hline
\end{tabular}

Here hyphens (-) indicates that the parameters cannot be found to satisfy conditions

depicts the histogram and quantile-quantile $(\mathrm{Q}-\mathrm{Q})$ plot in Fig. 1. We also applied various life distributions to fit the COVID-19 data set for the intention of comparative study. We have considered here the existing three models like odds Weibull distribution (OWD), Nadarajah-Haghighi distribution (NHD) and Exponentiated Nadarajah-Haghighi distribution (ENHD) for the same data. For more details please refer to [47].

Pdf and cdf of Weibull distribution are respectively

$$
f(x)=\left(\frac{\beta}{\alpha}\right)\left(\frac{x}{\alpha}\right)^{\beta-1} e^{-\left(\frac{x}{\alpha}\right)^{\beta}} ; x>0, \alpha>0, \beta>0
$$

and $F(x)=1-e^{-\left(\frac{x}{\alpha}\right)^{\beta}} ; x>0, \alpha>0, \beta>0$

Pdf and cdf of odds Weibull distribution (OWD) are respectively (suggested by [48])

$$
f(x)=\left(\frac{\alpha \beta}{x}\right)\left(\frac{x}{\theta}\right)^{\alpha} e^{\left(\frac{x}{\theta}\right)^{\alpha}}\left(e^{\left(\frac{x}{\theta}\right)^{\alpha}}-1\right)^{\beta-1}\left[1+\left(e^{\left(\frac{x}{\theta}\right)^{\alpha}}-1\right)^{\beta}\right]^{-2} ; x>0, \alpha
$$
and $F(x)=1-\left[1+\left(e^{\left(\frac{x}{\theta}\right)^{\alpha}}-1\right)^{\beta}\right]^{-1} ; \quad x>0, \quad \alpha<0$,
$0<(\beta, \theta)$.

Pdf and cdf of Nadarajah-Haghighi distribution (NHD) are respectively (see [49])

$$
f(x)=(\alpha \lambda)(1+\lambda x)^{\alpha-1} e^{1-(1+\lambda x)^{\alpha}} ; x>0, \alpha>0, \lambda>0
$$

and $F(x)=1-e^{1-(1+\lambda x)^{\alpha}} ; x>0, \alpha>0, \lambda>0$.

Pdf and cdf of Exponentiated Nadarajah-Haghighi distribution (ENHD) are respectively (see [49])

$$
f(x)=(\alpha \lambda \theta)(1+\lambda x)^{\alpha-1} e^{1-(1+\lambda x)^{\alpha}}\left(1-e^{1-(1+\lambda x)^{\alpha}}\right)^{\theta-1} ; x>0, \alpha>0, \lambda>0, \theta>0
$$

and $F(x)=\left(1-e^{1-(1+\lambda x)^{\alpha}}\right)^{\theta} ; x>0, \alpha>0, \lambda>0, \theta>0$. 
Table 8 Sample size comparison between the proposed plan and existing plan for $\tilde{a}=0.10 ; \beta=2$ and $a=1.0$

\begin{tabular}{|c|c|c|c|c|c|c|c|c|c|}
\hline \multirow[t]{2}{*}{$\tilde{\beta}$} & \multirow[t]{2}{*}{$\frac{\mu_{N}}{\mu_{0 N}}$} & \multicolumn{2}{|l|}{$I_{U}=0.00$} & \multicolumn{2}{|l|}{$I_{U}=0.02$} & \multicolumn{2}{|l|}{$I_{U}=0.04$} & \multicolumn{2}{|l|}{$I_{U}=0.05$} \\
\hline & & Proposed & Existing & Proposed & Existing & Proposed & Existing & Proposed & Existing \\
\hline 0.25 & 1.1 & 180.96 & 229 & 167.43 & 206 & 158.45 & 190 & 152.44 & 188 \\
\hline 0.25 & 1.2 & 55.27 & 65 & 49.78 & 60 & 48.58 & 59 & 48.38 & 56 \\
\hline 0.25 & 1.3 & 31.20 & 36 & 26.98 & 35 & 26.05 & 33 & 27.77 & 32 \\
\hline 0.25 & 1.4 & 20.21 & 25 & 18.62 & 23 & 17.60 & 22 & 18.00 & 18 \\
\hline 0.25 & 1.5 & 13.71 & 17 & 16.85 & 16 & 10.47 & 15 & 11.57 & 15 \\
\hline 0.25 & 1.8 & 6.95 & 11 & 8.00 & 11 & 10.82 & 10 & 8.23 & 9 \\
\hline 0.25 & 2.0 & 7.42 & 10 & 6.75 & 10 & 6.00 & 9 & 7.76 & 9 \\
\hline 0.10 & 1.1 & 263.63 & 371 & 250.98 & 352 & 233.82 & 324 & 220.09 & 317 \\
\hline 0.10 & 1.2 & 78.21 & 109 & 79.30 & 104 & 75.79 & 99 & 67.40 & 97 \\
\hline 0.10 & 1.3 & 43.98 & 56 & 36.43 & 55 & 34.72 & 51 & 36.26 & 51 \\
\hline 0.10 & 1.4 & 26.78 & 38 & 26.29 & 36 & 26.89 & 35 & 23.41 & 35 \\
\hline 0.10 & 1.5 & 19.27 & 30 & 19.59 & 28 & 19.95 & 27 & 18.92 & 24 \\
\hline 0.10 & 1.8 & 13.62 & 15 & 8.74 & 14 & 11.31 & 14 & 11.16 & 13 \\
\hline 0.10 & 2.0 & 10.25 & 13 & 8.74 & 13 & 9.66 & 12 & 9.05 & 9 \\
\hline 0.05 & 1.1 & 313.09 & 494 & 289.79 & 462 & 271.36 & 431 & 261.00 & 416 \\
\hline 0.05 & 1.2 & 88.23 & 146 & 91.16 & 138 & 80.65 & 129 & 78.19 & 119 \\
\hline 0.05 & 1.3 & 50.18 & 73 & 44.56 & 69 & 43.38 & 66 & 38.63 & 65 \\
\hline 0.05 & 1.4 & 33.51 & 49 & 30.35 & 44 & 27.18 & 42 & 28.07 & 41 \\
\hline 0.05 & 1.5 & 22.62 & 38 & 19.74 & 37 & 20.04 & 35 & 18.92 & 30 \\
\hline 0.05 & 1.8 & 13.24 & 22 & 12.52 & 21 & 13.51 & 20 & 11.65 & 18 \\
\hline 0.05 & 2.0 & 11.67 & 20 & 9.99 & 18 & 9.66 & 17 & 11.65 & 16 \\
\hline 0.01 & 1.1 & 405.20 & 229 & 370.60 & 206 & 349.70 & 190 & 345.60 & 188 \\
\hline 0.01 & 1.2 & 115.10 & 65 & 111.20 & 60 & 98.57 & 59 & 98.24 & 56 \\
\hline 0.01 & 1.3 & 60.36 & 36 & 54.33 & 35 & 52.56 & 33 & 49.45 & 32 \\
\hline 0.01 & 1.4 & 40.78 & 25 & 37.17 & 23 & 34.68 & 22 & 34.57 & 18 \\
\hline 0.01 & 1.5 & 29.01 & 17 & 30.69 & 16 & 24.68 & 15 & 25.42 & 15 \\
\hline 0.01 & 1.8 & 15.97 & 11 & 13.37 & 11 & 14.35 & 10 & 14.17 & 9 \\
\hline 0.01 & 2.0 & 16.25 & 10 & 13.07 & 10 & 11.13 & 9 & 10.62 & 9 \\
\hline
\end{tabular}

Table $\mathbf{9}$ COVID-19 data belong to Italy from 1 April to 20 July 2020

\begin{tabular}{llllllllll}
\hline $\mathbf{0 . 0 1 3 8}$ & $\mathbf{0 . 0 3 6 5}$ & $\mathbf{0 . 0 3 7 2}$ & $\mathbf{0 . 0 3 8 5}$ & $\mathbf{0 . 0 3 8 5}$ & $\mathbf{0 . 0 4 3 5}$ & $\mathbf{0 . 0 4 5 7}$ & $\mathbf{0 . 0 4 7 6}$ & $\mathbf{0 . 0 4 7 6}$ & $\mathbf{0 . 0 5 3 7}$ \\
\hline 0.0561 & 0.0562 & 0.0673 & 0.0769 & 0.0777 & 0.0802 & 0.0864 & 0.0870 & 0.0894 & 0.0942 \\
0.1041 & 0.1053 & 0.1071 & 0.1119 & 0.1149 & 0.1154 & 0.1176 & 0.1180 & 0.1221 & 0.1227 \\
0.1253 & 0.1264 & 0.1297 & 0.1302 & 0.1311 & 0.1319 & 0.1369 & 0.1375 & 0.1387 & 0.1390 \\
0.1398 & 0.1408 & 0.1417 & 0.1421 & 0.1443 & 0.1456 & 0.1491 & 0.1493 & 0.1520 & 0.1522 \\
0.1548 & 0.1593 & 0.1597 & 0.1619 & 0.1620 & 0.1628 & 0.1641 & 0.1646 & 0.1666 & 0.1686 \\
0.1730 & 0.1749 & 0.1754 & 0.1761 & 0.1767 & 0.1779 & 0.1789 & 0.1791 & 0.1827 & 0.1831 \\
0.1856 & 0.1915 & 0.1956 & 0.1957 & 0.1965 & 0.1987 & 0.1993 & 0.1994 & 0.1994 & 0.2003 \\
0.2012 & 0.2032 & 0.2057 & 0.2070 & 0.2113 & 0.2148 & 0.2167 & 0.2190 & 0.2195 & 0.2195 \\
0.2196 & 0.2212 & 0.2254 & 0.2321 & 0.2406 & 0.2421 & 0.2430 & 0.2495 & 0.2555 & 0.2641 \\
0.2667 & 0.2668 & 0.2690 & 0.2792 & 0.3067 & 0.3067 & 0.3176 & 0.3371 & 0.3436 & 0.3515 \\
0.4972 & & & & & & & & \\
\hline
\end{tabular}



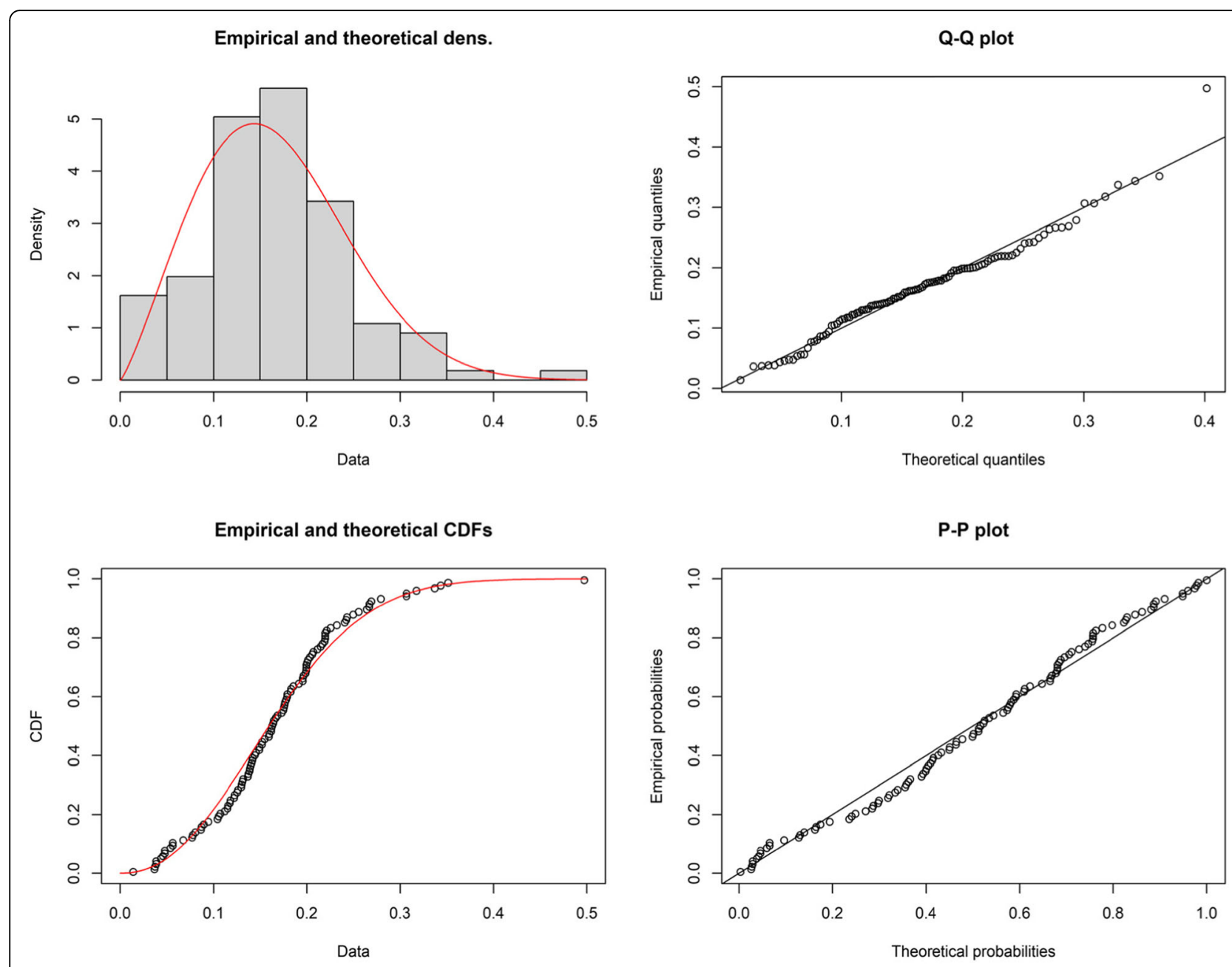

Fig. 1 The empirical and theoretical pdfs, empirical and theoretical cdfs, Q-Q plots and p-p plot for the WD for the daily new deaths

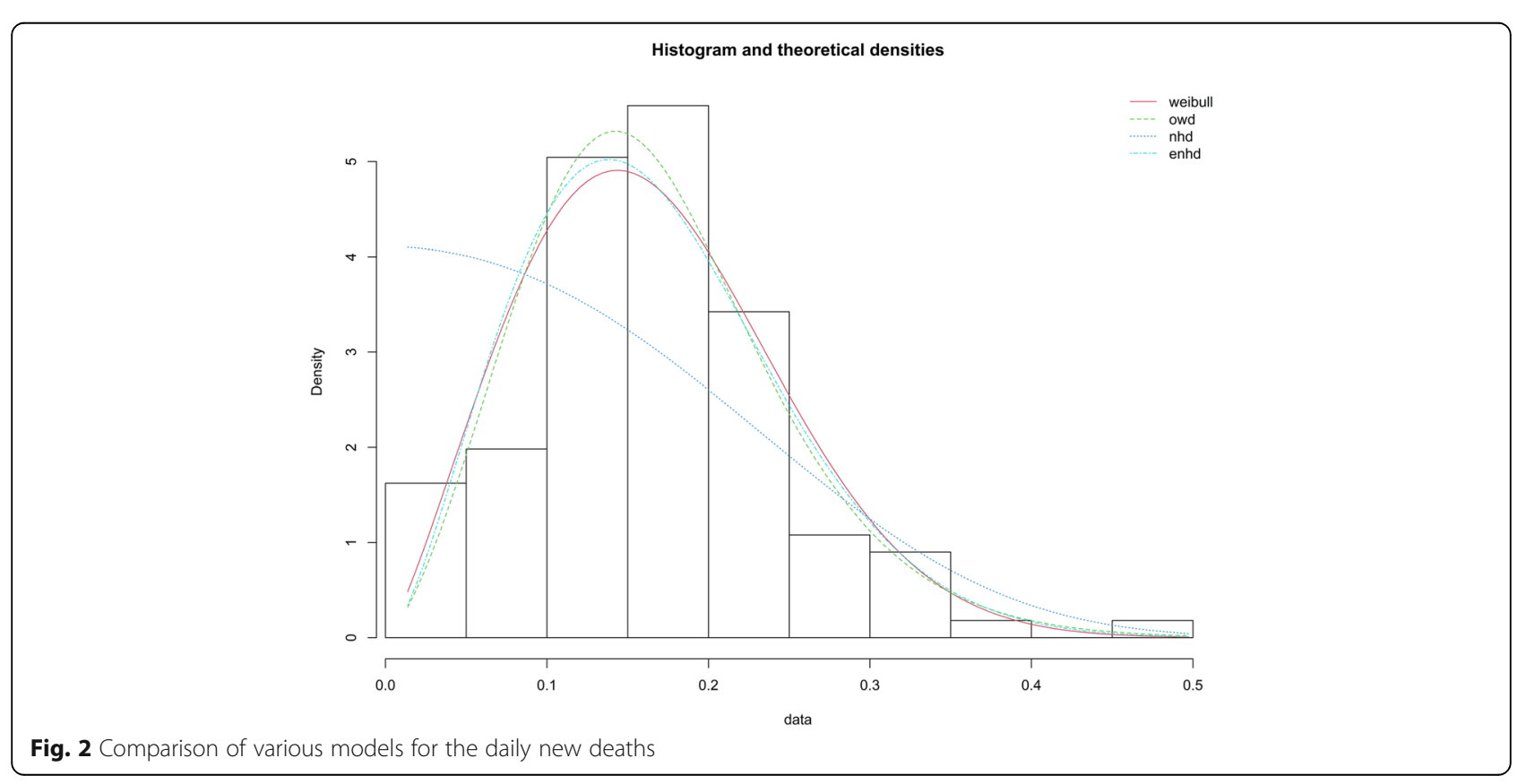


Table 10 Estimation and Goodness of fit measures of fitted distribution for daily new deaths

\begin{tabular}{|c|c|c|c|c|c|c|c|}
\hline \multirow{2}{*}{$\frac{\text { Dist }}{W D}$} & \multicolumn{3}{|c|}{ MLEs of the parameters } & \multirow{2}{*}{$\frac{\text { KS test } \boldsymbol{p} \text {-value }}{0.6766}$} & \multirow{2}{*}{$\frac{-2 \log L L}{-257.1131}$} & \multirow{2}{*}{$\frac{\text { AIC }}{-253.1131}$} & \multirow{2}{*}{$\frac{\text { BIC }}{-247.6940}$} \\
\hline & $\hat{a}=0.1880$ & $\hat{\beta}=2.2222$ & - & & & & \\
\hline OWD & $\hat{a}=1.7988$ & $\hat{\beta}=1.3225$ & $\hat{\theta}=0.1943$ & $2.2 \mathrm{e}-16$ & -258.5084 & -252.5084 & -244.3798 \\
\hline NHD & $\hat{a}=116.5132$ & $\hat{\lambda}=0.0353$ & & $8.549 \mathrm{e}-06$ & -221.1094 & -217.1095 & -211.6904 \\
\hline ENHD & $\hat{a}=3.7626$ & $\hat{\lambda}=1.6968$ & $\hat{\theta}=2.5732$ & 0.6324 & -256.9344 & -250.9344 & -242.8058 \\
\hline
\end{tabular}

We have estimated the parameters and good fit for the COVID-19 data for WD, OWD, NHD and ENHD, and are reported in Table 10 and depicted in Fig. 2. From Table 10 and Fig. 2 it is noticed that WD shows less AIC, BIC and -2logLL, moreover OWD and NHD are not fitted for COVID-19 data. Hence, Weibull distribution shows a good fit for the COVID-19 data belongs to
Italy. The plan parameters for this shape parameter are shown in Tables 11 and 12 . For the proposed plan, the shape parameter is $\hat{\beta}_{N}=(1+0.04) \times 2.2222 \approx 2.31$ when $I_{U}=0.04$.

Suppose that a quality medical practitioner would like to use the proposed repetitive sampling plan for Weibull

Table 11 The plan parameter when $\tilde{a}=0.10 ; \beta=2.2222$ and $a=0.5$

\begin{tabular}{|c|c|c|c|c|c|c|c|c|c|c|c|c|c|c|c|c|c|}
\hline \multirow[t]{2}{*}{$\tilde{\beta}$} & \multirow[t]{2}{*}{$\frac{\mu_{N}}{\mu_{O N}}$} & \multicolumn{4}{|c|}{$I_{U}=0.00$} & \multicolumn{4}{|c|}{$I_{U}=0.02$} & \multicolumn{4}{|c|}{$I_{U}=0.04$} & \multicolumn{4}{|c|}{$I_{U}=0.05$} \\
\hline & & $\mathbf{n}$ & $c_{1}$ & $c_{2}$ & ASN & $\mathrm{n}$ & $c_{1}$ & $c_{2}$ & ASN & $\mathrm{n}$ & $c_{1}$ & $c_{2}$ & ASN & $\mathrm{n}$ & $c_{1}$ & $c_{2}$ & ASN \\
\hline 0.25 & 1.1 & 312 & 40 & 47 & 507.20 & 287 & 39 & 46 & 471.79 & 305 & 45 & 51 & 447.47 & 256 & 38 & 45 & 428.73 \\
\hline 0.25 & 1.2 & 87 & 9 & 13 & 150.31 & 106 & 13 & 16 & 147.51 & 100 & 13 & 16 & 139.26 & 52 & 5 & 10 & 135.80 \\
\hline 0.25 & 1.3 & 60 & 6 & 8 & 80.77 & 57 & 6 & 8 & 76.36 & 60 & 7 & 9 & 79.08 & 41 & 4 & 7 & 72.91 \\
\hline 0.25 & 1.4 & 26 & 1 & 4 & 59.83 & 36 & 3 & 5 & 53.13 & 46 & 5 & 6 & 52.93 & 34 & 3 & 5 & 49.13 \\
\hline 0.25 & 1.5 & 37 & 3 & 4 & 43.78 & 21 & 1 & 3 & 36.92 & 31 & 3 & 4 & 37.42 & 19 & 1 & 3 & 33.81 \\
\hline 0.25 & 1.8 & 25 & 2 & 2 & 25.00 & 21 & 1 & 2 & 26.09 & 19 & 1 & 2 & 23.99 & 22 & 2 & 2 & 22.00 \\
\hline 0.25 & 2.0 & 27 & 2 & 2 & 27.00 & 20 & 1 & 2 & 25.33 & 10 & 0 & 1 & 14.66 & 11 & 0 & 1 & 15.30 \\
\hline 0.10 & 1.1 & 431 & 53 & 64 & 742.14 & 413 & 54 & 65 & 703.25 & 376 & 52 & 63 & 655.88 & 406 & 59 & 69 & 635.70 \\
\hline 0.10 & 1.2 & 140 & 14 & 20 & 232.64 & 143 & 16 & 21 & 208.70 & 110 & 12 & 18 & 195.90 & 114 & 13 & 19 & 195.07 \\
\hline 0.10 & 1.3 & 74 & 6 & 10 & 117.74 & 70 & 6 & 10 & 110.54 & 74 & 7 & 11 & 110.43 & 82 & 9 & 12 & 106.44 \\
\hline 0.10 & 1.4 & 47 & 3 & 6 & 73.54 & 44 & 3 & 6 & 69.36 & 51 & 4 & 7 & 72.13 & 48 & 4 & 7 & 70.57 \\
\hline 0.10 & 1.5 & 42 & 2 & 5 & 63.55 & 36 & 2 & 5 & 61.50 & 26 & 1 & 4 & 50.99 & 36 & 2 & 5 & 54.79 \\
\hline 0.10 & 1.8 & 18 & 0 & 2 & 31.17 & 26 & 1 & 3 & 38.16 & 16 & 0 & 2 & 27.66 & 25 & 1 & 3 & 35.16 \\
\hline 0.10 & 2.0 & 19 & 0 & 2 & 31.25 & 17 & 0 & 2 & 29.34 & 23 & 1 & 2 & 26.93 & 23 & 1 & 2 & 26.63 \\
\hline 0.05 & 1.1 & 507 & 61 & 75 & 883.90 & 544 & 71 & 84 & 836.31 & 486 & 67 & 80 & 773.35 & 472 & 67 & 80 & 752.06 \\
\hline 0.05 & 1.2 & 172 & 17 & 24 & 266.94 & 154 & 16 & 23 & 246.46 & 152 & 17 & 24 & 238.68 & 141 & 16 & 23 & 225.79 \\
\hline 0.05 & 1.3 & 99 & 8 & 13 & 146.61 & 94 & 8 & 13 & 136.91 & 80 & 7 & 12 & 124.05 & 78 & 7 & 12 & 120.03 \\
\hline 0.05 & 1.4 & 54 & 3 & 7 & 88.62 & 61 & 4 & 8 & 89.14 & 56 & 4 & 8 & 85.25 & 55 & 4 & 8 & 82.24 \\
\hline 0.05 & 1.5 & 53 & 3 & 6 & 71.76 & 51 & 3 & 6 & 67.35 & 46 & 3 & 6 & 63.79 & 32 & 1 & 5 & 62.18 \\
\hline 0.05 & 1.8 & 33 & 1 & 3 & 41.98 & 30 & 1 & 3 & 39.13 & 29 & 1 & 3 & 37.07 & 27 & 1 & 3 & 35.64 \\
\hline 0.05 & 2.0 & 23 & 0 & 2 & 31.96 & 21 & 0 & 2 & 29.89 & 19 & 0 & 2 & 27.97 & 20 & 0 & 2 & 27.50 \\
\hline 0.01 & 1.1 & - & - & - & - & - & - & - & - & - & - & - & - & - & - & - & - \\
\hline 0.01 & 1.2 & 204 & 18 & 29 & 341.70 & 192 & 18 & 29 & 321.80 & 207 & 22 & 32 & 298.90 & 216 & 24 & 34 & 297.80 \\
\hline 0.01 & 1.3 & 133 & 10 & 17 & 181.10 & 93 & 6 & 14 & 172.80 & 109 & 9 & 16 & 156.30 & 106 & 9 & 16 & 151.50 \\
\hline 0.01 & 1.4 & 87 & 5 & 11 & 127.20 & 84 & 5 & 11 & 117.10 & 69 & 4 & 10 & 106.60 & 58 & 3 & 9 & 99.95 \\
\hline 0.01 & 1.5 & 56 & 2 & 7 & 88.85 & 63 & 3 & 8 & 89.39 & 51 & 2 & 7 & 77.16 & 49 & 2 & 7 & 75.48 \\
\hline 0.01 & 1.8 & 43 & 1 & 4 & 53.38 & 47 & 1 & 5 & 59.58 & 39 & 1 & 4 & 47.41 & 37 & 1 & 4 & 45.83 \\
\hline 0.01 & 2.0 & 31 & 0 & 3 & 43.35 & 30 & 0 & 3 & 40.74 & 27 & 0 & 3 & 38.37 & 28 & 0 & 3 & 37.24 \\
\hline
\end{tabular}


Table 12 The plan parameter when $\tilde{a}=0.10 ; \beta=2.2222$ and $a=1.0$

\begin{tabular}{|c|c|c|c|c|c|c|c|c|c|c|c|c|c|c|c|c|c|}
\hline \multirow[t]{2}{*}{$\tilde{\beta}$} & \multirow{2}{*}{$\frac{\mu_{N}}{\mu_{0 N}}$} & \multicolumn{4}{|c|}{$I_{U}=0.00$} & \multicolumn{4}{|c|}{$I_{U}=0.02$} & \multicolumn{4}{|c|}{$I_{U}=0.04$} & \multicolumn{4}{|c|}{$I_{U}=0.05$} \\
\hline & & $n$ & $c_{1}$ & $c_{2}$ & ASN & $\mathrm{n}$ & $c_{1}$ & $c_{2}$ & ASN & $n$ & $c_{1}$ & $c_{2}$ & ASN & $n$ & $c_{1}$ & $c_{2}$ & ASN \\
\hline 0.25 & 1.1 & 113 & 55 & 59 & 151.82 & 86 & 43 & 48 & 136.48 & 80 & 42 & 47 & 130.72 & 73 & 39 & 44 & 122.99 \\
\hline 0.25 & 1.2 & 38 & 17 & 19 & 48.43 & 41 & 20 & 21 & 45.66 & 24 & 11 & 14 & 41.34 & 20 & 9 & 12 & 36.62 \\
\hline 0.25 & 1.3 & 14 & 5 & 7 & 21.80 & 15 & 6 & 8 & 23.22 & 21 & 10 & 11 & 24.55 & 14 & 6 & 8 & 21.94 \\
\hline 0.25 & 1.4 & 16 & 6 & 7 & 18.75 & 14 & 5 & 7 & 20.53 & 12 & 5 & 6 & 14.76 & 12 & 5 & 6 & 14.55 \\
\hline 0.25 & 1.5 & 9 & 3 & 4 & 11.62 & 11 & 4 & 5 & 13.48 & 5 & 1 & 3 & 12.11 & 6 & 2 & 3 & 8.28 \\
\hline 0.25 & 1.8 & 8 & 2 & 3 & 9.85 & 4 & 0 & 2 & 8.97 & 5 & 1 & 2 & 6.60 & 6 & 2 & 3 & 8.28 \\
\hline 0.25 & 2.0 & 3 & 0 & 1 & 4.60 & 6 & 2 & 2 & 6.00 & 6 & 1 & 2 & 7.05 & 5 & 1 & 2 & 6.48 \\
\hline 0.10 & 1.1 & 144 & 67 & 75 & 221.16 & 109 & 52 & 61 & 209.33 & 114 & 58 & 66 & 191.67 & 104 & 54 & 62 & 184.96 \\
\hline 0.10 & 1.2 & 44 & 18 & 22 & 64.25 & 44 & 19 & 23 & 62.91 & 38 & 17 & 21 & 56.83 & 35 & 16 & 20 & 54.71 \\
\hline 0.10 & 1.3 & 23 & 8 & 11 & 33.52 & 27 & 11 & 13 & 33.00 & 18 & 6 & 10 & 32.88 & 16 & 6 & 9 & 27.15 \\
\hline 0.10 & 1.4 & 18 & 6 & 8 & 23.33 & 17 & 6 & 8 & 22.23 & 11 & 3 & 6 & 20.57 & 16 & 6 & 8 & 20.58 \\
\hline 0.10 & 1.5 & 8 & 1 & 4 & 17.30 & 14 & 4 & 6 & 17.44 & 8 & 2 & 4 & 12.83 & 12 & 4 & 6 & 16.54 \\
\hline 0.10 & 1.8 & 9 & 2 & 3 & 10.36 & 4 & 0 & 2 & 8.97 & 10 & 3 & 4 & 11.40 & 8 & 2 & 3 & 9.12 \\
\hline 0.10 & 2.0 & 5 & 0 & 2 & 8.54 & 5 & 0 & 2 & 7.95 & 6 & 1 & 2 & 7.05 & 5 & 0 & 2 & 7.19 \\
\hline 0.05 & 1.1 & 178 & 82 & 92 & 262.01 & 168 & 82 & 91 & 240.57 & 126 & 63 & 73 & 220.31 & 123 & 63 & 73 & 217.07 \\
\hline 0.05 & 1.2 & 45 & 17 & 23 & 76.34 & 50 & 21 & 26 & 71.19 & 47 & 20 & 26 & 72.09 & 30 & 12 & 18 & 65.41 \\
\hline 0.05 & 1.3 & 33 & 12 & 15 & 40.86 & 26 & 9 & 13 & 37.70 & 18 & 6 & 10 & 32.88 & 24 & 9 & 13 & 35.44 \\
\hline 0.05 & 1.4 & 14 & 3 & 7 & 27.30 & 16 & 4 & 8 & 26.27 & 16 & 5 & 8 & 22.68 & 18 & 6 & 9 & 23.65 \\
\hline 0.05 & 1.5 & 13 & 3 & 6 & 20.78 & 10 & 2 & 5 & 18.00 & 12 & 3 & 6 & 18.41 & 12 & 3 & 6 & 17.53 \\
\hline 0.05 & 1.8 & 10 & 2 & 4 & 13.55 & 8 & 1 & 3 & 10.32 & 11 & 3 & 4 & 11.95 & 11 & 3 & 4 & 11.81 \\
\hline 0.05 & 2.0 & 8 & 1 & 3 & 10.95 & 7 & 1 & 3 & 10.57 & 9 & 2 & 3 & 9.82 & 6 & 1 & 2 & 6.95 \\
\hline 0.01 & 1.1 & 195 & 86 & 102 & 325.80 & 200 & 94 & 109 & 305.97 & 214 & 107 & 121 & 292.44 & 185 & 94 & 108 & 275.80 \\
\hline 0.01 & 1.2 & 69 & 26 & 34 & 95.71 & 59 & 23 & 31 & 88.26 & 54 & 22 & 30 & 83.99 & 63 & 27 & 35 & 85.42 \\
\hline 0.01 & 1.3 & 38 & 12 & 18 & 52.35 & 29 & 9 & 15 & 46.76 & 32 & 11 & 17 & 46.48 & 27 & 9 & 15 & 43.28 \\
\hline 0.01 & 1.4 & 21 & 5 & 10 & 33.36 & 20 & 5 & 10 & 31.56 & 19 & 5 & 10 & 30.11 & 19 & 5 & 10 & 28.11 \\
\hline 0.01 & 1.5 & 21 & 4 & 9 & 27.10 & 14 & 2 & 7 & 24.13 & 16 & 4 & 8 & 23.27 & 14 & 3 & 7 & 19.98 \\
\hline 0.01 & 1.8 & 16 & 3 & 5 & 16.99 & 10 & 1 & 4 & 13.08 & 11 & 2 & 4 & 12.32 & 9 & 1 & 4 & 12.15 \\
\hline 0.01 & 2.0 & 10 & 1 & 3 & 11.31 & 7 & 0 & 3 & 11.04 & 6 & 0 & 3 & 11.45 & 9 & 1 & 3 & 9.93 \\
\hline
\end{tabular}

distribution under indeterminacy to ensure the mean ratio of daily new deaths at least 60 days using the truncated life test for 60 days. Let the producer's risk be $10 \%$ at $\mu_{N} / \mu_{0 N}=1.1$ and the consumer's risk is $10 \%$. From Table 11, with $\mathrm{a}=1.0, \tilde{\beta}=0.10$ and $\tilde{\alpha}=0.10$ for the repetitive sampling plan, it could be found that the plan parameters are $c_{1}=58 c_{2}=66$ and ASN $=191.67$. Therefore, the plan could be implemented as follows: selecting a random sample of 114 patients from the arrived lot of patients, and doing the truncated life test for 60 days. The proposed sampling plan will be implemented as: accept the null hypothesis $H_{0}: \mu_{N}=0.1665$ if the average ratio of daily new deaths in 60 days is less than 58 , the ratio of daily deaths, but the lot should be rejected as soon as the ratio of daily new deaths exceeds 66 . Otherwise, the experiment would be repeated. Table 9 shows the 56 ratios of daily new deaths before the average ratio of daily new deaths of 0.1665 . Therefore, the quality medical practitioners would have accepted the arrived lot of patients.

\section{Conclusions}

An elaborated investigation of COVID-19 data for Weibull distribution under indeterminacy using time truncated repetitive sampling plan is studied. The proposed design parameters are obtained for known values of the indeterminacy parameters. The plan parameters and corresponding tables are developed for the industrial purposes at specified indeterminacy parametric values. The proposed sampling plan is compared with the existing sampling plans. The result shows that the proposed repetitive sampling plan is more economical than the existing sampling plan. The proposed sampling plan 
saves time; labor and amount for experimentation, the proposed plan is recommended to apply for testing the average number of deaths due to COVID-19. Also, noticed that if the indeterminacy values increase then the average sample number is decreased. The developed repetitive sampling plan procedure is illustrated with COVID-19 data belong to Italy as an application. The proposed sampling plan can be implemented in various industries covering the packing industry, medical sciences, food industries and electronic industries. Further research can be established to extend our study to group sampling plans, multiple dependent state sampling plans, and multiple dependent state repetitive sampling plans.

\section{Abbreviations}

ASN: Average sample number; RASP: Repetitive acceptance sampling plan; OC: Operating characteristic; Q-Q: Quantile-quantile

\section{Acknowledgements}

The authors are deeply thankful to the editor and reviewers for their valuable suggestions to improve the quality of the paper.

\section{Authors' contributions}

G.S.R and M. A wrote the paper. All authors have read and approved the manuscript.

\section{Funding}

None.

\section{Availability of data and materials}

The data is given in the paper

\section{Declarations}

Ethics approval and consent to participate

$\mathrm{N} / \mathrm{A}$

\section{Consent for publication}

N/A

\section{Competing interests}

None.

\section{Author details}

'Department of Statistics, University of Dodoma, PO. Box: 259, Dodoma, Tanzania. ${ }^{2}$ Department of Statistics, Faculty of Science, King Abdulaziz University, Jeddah 21551, Saudi Arabia.

Received: 28 June 2021 Accepted: 6 September 2021

Published online: 25 October 2021

\section{References}

1. Mizumoto $\mathrm{K}$, et al. Estimating the asymptomatic proportion of coronavirus disease 2019 (COVID-19) cases on board the Diamond Princess cruise ship Yokohama, Japan, 2020. Euro Surveill. 2020:25(10):2000180.

2. Hogan CA, Sahoo MK, Pinsky BA. Sample pooling as a strategy to detect community transmission of SARS-CoV-2. JAMA. 2020;323(19):1967-9.

3. Kantam RRL, Rosaiah K, Rao GS. Acceptance sampling based on life tests: log-logistic model. J Appl Stat. 2001;28(1):121-8.

4. Tsai T-R, Wu S-J. Acceptance sampling based on truncated life tests for generalized Rayleigh distribution. J Appl Stat. 2006;33(6):595-600.

5. Balakrishnan N, Leiva V, López J. Acceptance sampling plans from truncated life tests based on the generalized Birnbaum-Saunders distribution. Commun Stat Simul Comput. 2007:36(3):643-56.

6. Lio YL, Tsai T-R, Wu S-J. Acceptance sampling plans from truncated life tests based on the Birnbaum-Saunders distribution for percentiles. Commun Stat Simul Comput. 2009;39(1):119-36.
7. Lio $Y L, T$ sai $T-R$, Wu S-J. Acceptance sampling plans from truncated life tests based on the Burr type XII percentiles. J Chin Inst Ind Eng. 2010; 27(4):270-80.

8. Al-Omari A, Al-Hadhrami S. Acceptance sampling plans based on truncated life tests for Extended Exponential distribution. Kuwait J Sci. 2018;45(2):30-41.

9. Al-Omari Al. Time truncated acceptance sampling plans for generalized inverted exponential distribution. Electron J Appl Stat Anal. 2015;8(1):1-12.

10. Yan A, Liu S, Dong X. Variables two stage sampling plans based on the coefficient of variation. J Adv Mech Des Syst Manuf. 2016;10(1):1-12.

11. Yen $\mathrm{C}-\mathrm{H}$, et al. A rectifying acceptance sampling plan based on the process capability index. Mathematics. 2020;8(1):141.

12. Aslam $\mathrm{M}$, et al. Decision rule of repetitive acceptance sampling plans assuring percentile life. Sci Iran. 2012;19(3):879-84.

13. Sherman RE. Design and evaluation of a repetitive group sampling plan. Technometrics. 1965;7(1):11-21.

14. Aslam M, Lio YL, Jun C-H. Repetitive acceptance sampling plans for burr type XII percentiles. Int J Adv Manuf Technol. 2013;68(1):495-507.

15. Aslam M, Azam M, Jun C-H. Decision rule based on group sampling plan under the inverse Gaussian distribution. Seq Anal. 2013;32(1):71-82.

16. Singh N, Singh N, Kaur $\mathrm{H}$. A repetitive acceptance sampling plan for generalized inverted exponential distribution based on truncated life test. Int J Sci Res Math Stat Sci. 2018;5(3):58-64.

17. Yan A, Liu S. Designing a repetitive group sampling plan for Weibull distributed processes. Math Probl Eng. 2016;2016:5862071.

18. Aslam $M$, et al. Designing of a new monitoring t-chart using repetitive sampling. Inf Sci. 2014;269:210-6.

19. Yen $\mathrm{C}-\mathrm{H}$, Chang $\mathrm{C}-\mathrm{H}$, Aslam M. Repetitive variable acceptance sampling plan for one-sided specification. J Stat Comput Simul. 2015;85(6):1102-16.

20. Smarandache F. Neutrosophy. Neutrosophic Probability, Set, and Logic, ProQuest Information \& Learning, vol. 105. Ann Arbor: American Research Press; 1998. p. 118-23

21. Smarandache, F. and H.E. Khalid, Neutrosophic precalculus and neutrosophic calculus 2015: Infinite Study.

22. Peng X, Dai J. Approaches to single-valued neutrosophic MADM based on MABAC, TOPSIS and new similarity measure with score function. Neural Comput \& Applic. 2018:29(10):939-54.

23. Abdel-Basset $M$, et al. Cosine similarity measures of bipolar neutrosophic set for diagnosis of bipolar disorder diseases. Artif Intell Med. 2019;101:101735.

24. Nabeeh NA, et al. An integrated neutrosophic-topsis approach and its application to personnel selection: a new trend in brain processing and analysis. IEEE Access. 2019;7:29734-44.

25. Pratihar J, et al. Transportation problem in neutrosophic environment. In: Neutrosophic Graph Theory and Algorithms. Hershey: IGl Global; 2020. p. 180-212.

26. Pratihar J, et al. Modified Vogel's approximation method for transportation problem under uncertain environment. Complex Intell Syst. 2020:1-12. in press. https://doi.org/10.1007/s40747-020-00153-4.

27. Smarandache, F., Introduction to neutrosophic statistics 2014: Infinite Study.

28. Chen J, Ye J, Du S. Scale effect and anisotropy analyzed for neutrosophic numbers of rock joint roughness coefficient based on neutrosophic statistics. Symmetry. 2017;9(10):208.

29. Chen J, et al. Expressions of rock joint roughness coefficient using neutrosophic interval statistical numbers. Symmetry. 2017:9(7):123.

30. Aslam M. A new failure-censored reliability test using neutrosophic statistical interval method. Int J Fuzzy Syst. 2019:21(4):1214-20.

31. Aslam M. A new sampling plan using Neutrosophic process loss consideration. Symmetry. 2018;10(5):132.

32. Aslam M. Design of Sampling Plan for exponential distribution under Neutrosophic statistical interval method. IEEE Access. 2018;6:64153-8.

33. Aslam M. A new attribute sampling plan using neutrosophic statistical interval method. Complex Intell Syst. 2019;5(4):365-70.

34. Aslam M, et al. Time-truncated group plan under a Weibull distribution based on Neutrosophic statistics. Mathematics. 2019;7(10):905

35. Alhasan, K.F.H. and F. Smarandache, Neutrosophic Weibull distribution and Neutrosophic Family Weibull Distribution 2019: Infinite Study.

36. Jamkhaneh EB, Sadeghpour-Gildeh B, Yari G. Important criteria of rectifying inspection for single sampling plan with fuzzy parameter. Int J Contemp Math Sci. 2009:4(36):1791-801.

37. Jamkhaneh EB, Sadeghpour-Gildeh B, Yari G. Inspection error and its effects on single sampling plans with fuzzy parameters. Struct Multidiscip Optim. 2011;43(4):555-60. 
38. Sadeghpour Gildeh B. E. Baloui Jamkhaneh, and G. Yari, acceptance single sampling plan with fuzzy parameter. Iran J Fuzzy Syst. 2011;8(2):47-55.

39. Afshari R, Sadeghpour Gildeh B. Designing a multiple deferred state attribute sampling plan in a fuzzy environment. Am J Math Manag Sci. 2017;36(4):328-45.

40. Tong $X$, Wang Z. Fuzzy acceptance sampling plans for inspection of geospatial data with ambiguity in quality characteristics. Comput Geosci. 2012:48:256-66.

41. Uma G, Ramya K. Impact of fuzzy logic on acceptance sampling plans-a review. Automation Autonomous Syst. 2015;7(7):181-5.

42. Aslam M. Testing average wind speed using sampling plan for Weibull distribution under indeterminacy. Sci Rep. 2021;11(1):7532.

43. Balamurali S, Jun C-H. Repetitive group sampling procedure for variables inspection. J Appl Stat. 2006;33(3):327-38.

44. Aslam M, Yen C-H, Jun C-H. Variable repetitive group sampling plans with process loss consideration. J Stat Comput Simul. 2011;81(11):1417-32.

45. Aslam $M$, et al. Developing a variables repetitive group sampling plan based on process capability index C pk with unknown mean and variance. J Stat Comput Simul. 2013;83(8):1507-17.

46. Hassan A-S, Almetwally E-M, Ibrahim G-M. Kumaraswamy Inverted ToppLeone Distribution with Applications to COVID-19 Data. Comput Mater Continua. 2021:68(1):337-58.

47. Lemonte AJ. A new exponential-type distribution with constant, decreasing, increasing, upside-down bathtub and bathtub-shaped failure rate function. Comput Stat Data Anal. 2013;62:149-70.

48. Cooray K. Generalization of the Weibull distribution: the odd Weibull family. Stat Model. 2006;6(3):265-77.

49. Alhussain ZA, Ahmed EA. Estimation of exponentiated Nadarajah-Haghighi distribution under progressively type-II censored sample with application to bladder cancer data. Indian J Pure Appl Math. 2020;51(2):631-57.

\section{Publisher's Note}

Springer Nature remains neutral with regard to jurisdictional claims in published maps and institutional affiliations.

Ready to submit your research? Choose BMC and benefit from:

- fast, convenient online submission

- thorough peer review by experienced researchers in your field

- rapid publication on acceptance

- support for research data, including large and complex data types

- gold Open Access which fosters wider collaboration and increased citations

- maximum visibility for your research: over $100 \mathrm{M}$ website views per year

At $\mathrm{BMC}$, research is always in progress.

Learn more biomedcentral.com/submissions 